J. Product. \& Dev., 22(1): 39-63(2017)

\title{
CHEMICAL AND MICROBIOLOGICAL STUDIES ON AQUEOUSEXTRACT OF MORINGA LEAVES AND ITS EFFECT ON FRUIT JUICE
}

\author{
A.M. El-Shawaf ${ }^{\prime}$; El-zainy, A.R.M. ${ }^{2}$; Fatma M. El-Zamzamy ${ }^{2}$ and \\ Nabila Awad, M.S.A.M. ${ }^{2}$ \\ 1-Department of Food and Dairy Science Faculty of Technology and \\ Development, Zagazig University, Egypt \\ 2-Department of Nutrition and Food Science, Faculty of Specific Education, \\ Mansoura University, Egypt
}

\begin{abstract}
The aim of this investigation is to study the effect of Moringa aqueous (MA) leaves extract addition to fresh fruit juices (pomegranate, strawberry, cantaloupe and white guava) on chemical, microbiological, physical and sensory properties. Moringa aqueous extract has high functional value for its high content of total carotenoids, vitamins; phenolic compounds and mineral show ever fruits have high sensory properties compared to moringa. Fresh fruit juices (guava pomegranate - cantaloupe - strawberry) and MA extract were mixed in equal proportions of 1:1. Chemical and microbial properties of drinks were evaluated.
\end{abstract}

Results revealed that high moisture content was for cantaloupe (89.84\%),followed by pomegranate juice (87.91\%), while Moringa aqueous extract recorded the lowest value $(85.01 \%)$ after the addition of aqueous extract of moringa to juice ratio of $1: 1$ increased moisture of pomegranate juice + MA extract (93.34\%), followed by cantaloupe + Moringa aqueous extract(92.05\%), while MA extract less content of moisture (85.01\%).Total Sugar in pomegranate juice (9.57\%), guava (9.42\%), while dry MA extract (4.95\%), whereas when adding of MA extract have a ratio of $1: 1$ record MA extract+ pomegranate juice (8.02\%), guava juice + MA extract $7.91 \%)$, and the lowest value $(4.95 \%)$ to MA extract. Minerals in juices increased after the addition of MA extract have a ratio of 1: 1 for example, calcium to pomegranate juice (20 mg / 100g), guava (17 mg / $100 \mathrm{~g}$ ), while after the addition of MA extract, ratio of 1: 1 record pomegranate juice + MA extract $(25.51 \mathrm{mg} /$ $100 \mathrm{~g}$ ), guava juice + MA extract (29.05 $\mathrm{mg} / 100 \mathrm{~g}$ ), and ratio increased for potassium, magnesium, iron and Zinc. high proportion of antioxidants juice increased after adding moringa ratio of 1: 1 ratio of 
carotenoids of guava juice $(21.74 \mathrm{mg} / 100 \mathrm{~g})$, cantaloupe juice $(20.20 \mathrm{mg}$ / 100g), strawberry juice (20.08 mg / $100 \mathrm{~g}$ ), while adding MA extract ratio of 1: 1 ratio of carotenoids: guava juice + MA extract(25.43 mg / $100 \mathrm{gm}$ ), strawberry juice + MA extract(24.12 mg / $100 \mathrm{~g})$.

Sensory evaluation increased susceptibility to MA extract after adding juice ratio of 1: 1 MA extract(84.68\%), strawberry juice + MA extract (90.5\%), cantaloupe juice + MA extract (89.82\%), and pomegranate juice + MA $(89.43 \%)$ guava juice + MA extract $(87.34 \%)$.

Microbial evaluation indicated a decline in microbial count of bacteria, mould and yeast in the moringa fruit aqueous extract during storage period of 15 days in refrigerator.

Conclusively, from obtained results, it can strengthen the Moringa aqueous extract with fruit juices to raise the nutritional value and as an antioxidant and antimicrobial (as a natural preservative).

Keywords: Moringa aqueous extract, antioxidant, antimicrobial, Microbial evaluation.

\section{INTRODUCTION}

Moringa leaves contain amount of calcium equal to 4 calcium existing in milk also contains potassium three times the existing in bananas contain magnesium 36 times that of wheat, and contain iron 25 times the spinach, contain chlorophyll four times the wheatgrass contains Vitamin C 7 times the existing in oranges. The leaves also very rich in fatty acids such as omega 3 , omega 6 and 9.omega, and a lot of other minerals and vitamins. Mahamood et al., (2010).

Moringa contains vitamin B 4 times its content found meat red and contain vitamin B1250 times that found in sardines. Itcontains vitamin B3 50 times that inpeanut and 3 times ofvitamin $\mathrm{E}$ found in almonds moringa leaves contain 2 double the protein found in yogurt and milk. The moringa leaves contain eight kinds of vitamins, 9 kinds of salts and minerals, 18 amino acid, 46 anti-oxidant substance of very high anti-bacteria and viruses material and 36 anti-inflammatory substance. Al-Dabbas et al., (2010). Moringa is a good tree taken advantage of all their parts (leaves and branches and peel the stem and fruit and roots), and most use are leaves. It is one of the fastest growing trees in the world. They contain the amount of calcium, potassium, Magnesium, Iron, Chlorophyll, vitamin C and Vitamin A. The leaves also very rich in fatty acids Fakurazi et al., (2012).Strawberries guava, pomegranate, and cantaloupe are a common and important fruits because of their high content of essential nutrients and beneficial phytochemicals, which 
seem to have relevant biological activity in human health. Among these phytochemicals, anthocyanin and ellagitannins are the major antioxidant compounds. Giampieri et al., (2012).

Therefore, this research aims to produce moringa with fruit juices mix adding obtain a functional valuerepresenting as an antioxidant, antimicrobial and a natural preservative.

\section{MATERIALS AND METHODS}

\section{Materials:}

Combination of plant material: Fresh leaves of $M$. oleifera Lam was collected from agriculture researches center at Dokki, Giza, Egypt, In November (2015), groups of plants were brought to the laboratory, thoroughly washed in running tap water to remove debris and dust particles and then rinsed in distilled water for five minutes, we dried green leaves in a shade under $28 \pm 2^{\circ} \mathrm{C}$ for 6 to 7 days and then crushed into coarse powdery substance by using electric grinder (Moulinex model No T27/v96). It was dried again and was then sieved to get fine powder using the fine plastic sieve that was stored in an air tight bottle in the laboratory until required.

Fresh fruits: Strawberry, white guava, pomegranate, and cantaloupe fruits were obtained from shops selling fruit in Meet-Ghamr city Dakahlia Governorate, Egypt.

Culture media: Nutrient Broth (M002) and Mac-Conkey Agar (w/0.15\%Bilts, $\mathrm{CV}$ and NaCI M081) were obtained from Algamhoria Company Cairo.

\section{Methods:}

Preparation of different Juice: Fresh fruit were washed, sorted, and cut up (40 grams), then $10 \mathrm{ml}$ of water were added to it and hitting by the blender then drained and then hurt (Jasmine, 2012).

The proportion of extracted juice $=-\begin{aligned} & \text { Weight of juice } \\ & \text { Weight of fruit }\end{aligned}$

Preparation of Moringa aqueous extract: Moringa aqueous extract was prepared by grinding moringa dry leaves $(50 \mathrm{ml}$ of boiled water were added to 4 grams of moringa powder left for five minutes and drained).

Preparation of moringa Juice drinks: Moringa juice drink (8 grams moringa $+50 \mathrm{ml}$ of boiled water) was fruit juice at ratio of $1: 1$. 
Chemical analysis: Moisture, ash, crude fiber, crude protein and fat,energyvalue,total soluble solids, Totalacidity, pHvalue, andminerals content were determined according to A.OA.C (2005).

Determination of sugars: Total reducing and non-reducing sugars were determined according to the Methods of Smith et al., (1999).

Determination of total carotenoids (as Total $\beta$ - carotene): Carotenoids were assessed as described by Wettestein (1997).

Determination of ascorbic acid: Ascorbic acid was fixed by using 2, 6 dichlorophenol indophenol titration method as qualified by Ranganna, (1999).

Determination of total phenol content: Total phenols content was determined using the FolinCiocaltea reagent (Singleton and Rossi, 2005).

\section{Determination of Vitamin E;:}

Vitamin E content in the samples was determined according to the methods of Gimeno,et al., (2000)

Determination of Total antioxidant: Total antioxidant and Total Flavonoid Content were determined according to Lallianrawna et al., (2013).

Microbiological examination: Total plate counts were carried out by applying the methods of Warakaulle et al., (2014).

Statistical Analysis: The experimental data were statistically analyzed using the analysis of variance by the factorial design method, this has been done to certain weather the differences between them are significant according to (Steel and Torrie, 1980).

Sensory evaluation: The final formulated mixtures were sensory evaluated by panel tests (panelists).According to Notter et al., (1995). Ten controllers were requested to evaluate the formulated mixtures they was asked to score all the organoleptic qualities in numerical system. The examined characteristics were appearance, texture, color, odor, flavor and taste.

\section{RESULTS AND DISCUSSION}

Data in Table (1) showed that the dressing percentage for juice was at the following decreasing order: strawberry $(88.2 \%)$, guava $(60 \%)$, cantaloupe $(50 \%)$ and pomegranate $(40.5 \%)$. 
Table (1): The proportion of extracted juice

\begin{tabular}{|l|c|c|c|}
\hline \multicolumn{1}{|c|}{ Samples } & $\begin{array}{c}\text { Weight of } \\
\text { fruit } \mathbf{( k g )}\end{array}$ & $\begin{array}{c}\text { Weight of juice } \\
\text { (kg) }\end{array}$ & $\begin{array}{c}\text { The proportion of } \\
\text { extracted juice (\%) }\end{array}$ \\
\hline Strawberry & 17 & 15 & 88.23 \\
\hline Guava & 25 & 15 & 60 \\
\hline Pomegranate & 37 & 15 & 40.54 \\
\hline Cantaloupe & 30 & 15 & 50 \\
\hline
\end{tabular}

Chemical composition of fresh leaves of moringa and natural fruits (W.W):

Moisture, ash, total carbohydrates, fiber, protein, fat and energy of the fresh moringa, fruits of strawberry, guava, pomegranate and cantaloupe contents are presented in Table 2. The data showed that there were significant differences $(\mathrm{P}<0.05)$. In moisture content as the highest content of moisture was in cantaloupe $(85.79 \%)$, followed by strawberry $(84.95 \%)$, guava $(84.3 \%)$, pomegranate $(84.10 \%)$, while the lowest content was in fresh moringa ( $81.85 \%)$. These results agree with those obtained by Coppin, (2014).Data showed that strawberry contains the highest amount of ash $(5.40 \%)$, followed by pomegranate $(3.65 \%)$, cantaloupe $(2.89 \%)$, fresh moringa $(2.50 \%)$, whereas guava has the lowest value $(1.13 \%)$, these results go in agreement with those found by Gaikwad et al., (2011).

In addition, data indicates that pomegranate contain the highest content of total carbohydrates $(6.31 \%)$, followed by guava $(6.84 \%)$, however the lowest was in fresh moringa (5.92\%), these results goin agreement with those found by Shahriar, et al., (2012).Table (2) registered that the highest fiber content was $4.02 \%$ in guava, followed by pomegranate (3.16\%), fresh moringa $(3.01 \%)$, strawberry $(2.30 \%)$, while the lowest was $2.08 \%$ for cantaloupe, which agree with the finding of Tanwar, et al., (2014).

On the other hand, the data illustrated in Table (2) showed that the highest protein content was observed in fresh moringa (3.9\%), followed by Pomegranate $(2.62 \%)$, guava $(2.55 \%)$, cantaloupe $(1.83 \%)$, whereas strawberry had the lowest content $(1.67 \%)$, which agree with the finding of Miano et al., (2010). Fat registered the highest content in fresh moringa 
Table (2): Some chemical composition of fresh leaves of moringa and natural fruits (strawberry, guava, pomegranate and cantaloupe) (W.W)

\begin{tabular}{|c|c|c|c|c|c|c|c|}
\hline $\begin{array}{c}\text { Content. } \\
\begin{array}{c}\text { Sample of } \\
\text { blends }\end{array}\end{array}$ & $\begin{array}{c}\text { Moisture } \\
\%\end{array}$ & $\begin{array}{c}\text { Ash } \\
\%\end{array}$ & $\begin{array}{c}\text { Total } \\
\text { Carbohydrates } \\
\%\end{array}$ & $\begin{array}{c}\text { Fiber } \\
\%\end{array}$ & $\begin{array}{c}\text { Protein } \\
\%\end{array}$ & $\begin{array}{c}\text { Fat } \\
\%\end{array}$ & $\begin{array}{c}\text { Energy } \\
\text { (Kcal) }\end{array}$ \\
\hline $\begin{array}{l}\text { Fresh leaves } \\
\text { of moringa }\end{array}$ & $\begin{array}{l}82.85^{\mathrm{c}} \\
\pm 0.03\end{array}$ & $\begin{array}{l}2.50^{\mathrm{c}} \\
\pm 0.00\end{array}$ & $\begin{array}{l}5.92^{\mathrm{d}} \\
\pm 0.00\end{array}$ & $\begin{array}{l}3.01^{\mathrm{b}} \\
\pm 0.01\end{array}$ & $\begin{array}{c}3.9^{\mathrm{a}} \\
\pm 0.07\end{array}$ & $\begin{array}{r}1.82^{\mathrm{a}} \\
\pm 0.01\end{array}$ & $\begin{array}{l}41.16^{\mathrm{a}} \\
\pm 0.31\end{array}$ \\
\hline Strawberry & $\begin{array}{c}84.95^{\mathrm{b}} \\
\pm 0.31\end{array}$ & $\begin{array}{l}5.40^{\mathrm{a}} \\
\pm 0.41\end{array}$ & $\begin{array}{l}5.08^{\mathrm{d}} \\
\pm 0.20\end{array}$ & $\begin{array}{l}2.30^{\mathrm{c}} \\
\pm 0.13\end{array}$ & $\begin{array}{l}1.67^{\mathrm{c}} \\
\pm 0.05\end{array}$ & $\begin{array}{l}0.60^{b} \\
\pm 0.00\end{array}$ & $\begin{array}{l}32.4^{\mathrm{c}} \\
\pm 0.32\end{array}$ \\
\hline Guava & $\begin{array}{l}84.3^{\mathrm{b}} \\
\pm 0.00\end{array}$ & $\begin{array}{l}2.13^{\mathrm{d}} \\
\pm 0.67\end{array}$ & $\begin{array}{l}6.84^{\mathrm{b}} \\
\pm 0.01\end{array}$ & $\begin{array}{l}4.02^{\mathrm{a}} \\
\pm 0.71\end{array}$ & $\begin{array}{l}2.55^{\mathrm{b}} \\
\pm 0.73\end{array}$ & $\begin{array}{l}0.16^{\mathrm{d}} \\
\pm 0.04\end{array}$ & $\begin{array}{c}39^{\mathrm{b}} \\
\pm 0.45\end{array}$ \\
\hline Pomegranate & $\begin{array}{c}84.10^{\mathrm{bc}} \\
\pm 0.12\end{array}$ & $\begin{array}{l}3.65^{\mathrm{b}} \\
\pm 0.03\end{array}$ & $\begin{array}{l}6.31^{\mathrm{a}} \\
\pm 0.10\end{array}$ & $\begin{array}{l}3.16^{6} \\
\pm 0.20\end{array}$ & $\begin{array}{l}2.62^{\mathrm{b}} \\
\pm 0.37\end{array}$ & $\begin{array}{l}0.16^{\mathrm{d}} \\
\pm 0.91\end{array}$ & $\begin{array}{l}37.28^{\mathrm{a}} \\
\pm 0.81\end{array}$ \\
\hline Cantaloupe & $\begin{array}{l}85.79^{\mathrm{a}} \\
\pm 0.21\end{array}$ & $\begin{array}{l}2.89^{\mathrm{b}} \\
\pm 0.51\end{array}$ & $\begin{array}{l}6.17^{\mathrm{c}} \\
\pm 0.23\end{array}$ & $\begin{array}{l}2.08^{\mathrm{c}} \\
\pm 0.04\end{array}$ & $\begin{array}{l}1.83^{\mathrm{c}} \\
\pm 0.01\end{array}$ & $\begin{array}{l}0.24^{\mathrm{c}} \\
\pm 0.19\end{array}$ & $\begin{array}{l}34.16^{\mathrm{c}} \\
\pm 0.93\end{array}$ \\
\hline
\end{tabular}

a,b,c Mean in the same row are bearing letters, differed significantly at $(\mathrm{P}<0.05)$.

$(1.82 \%)$, guava, $(0.95 \%)$, strawberry $(0.60 \%)$, cantaloupe $(0.24 \%)$, while the lowest value was $0.16 \%$ for pomegranate which agrees with the finding of Mahendran (2010). The highest value was $41.16 \mathrm{kcal}$ in fresh moringa, followed by $39 \mathrm{kcal}$ in guava, $37 \mathrm{kcal}$ in pomegrante, $34.2 \mathrm{kcal}$ in cantaloupe, and the lowest value was $32.4 \mathrm{kcal}$ in strawberry.

Chemical composition of fresh fruit juice strawberry, guava, pomegranate and cantaloupe, fresh and dry leaves of moringa aqueous extract $(W . W)$ :

Moisture, ash, total carbohydrates, fiber, protein, fat and food energy of the fresh Moringa aqueous (MA) extract, dry MA extract, strawberry, guava, pomegranate and cantaloupe contents are presented in Table (3). The data tabulated in Table (3) showed that there were significant differences $(\mathrm{P}<0.05)$. The highest content of moisture was in cantaloupe juice $(89.48 \%)$, followed by pomegranate $(87.91 \%)$, while the lowest content was in dry MA extract $(85.01 \%)$. These results agree with those obtained by El-Massry et al., (2013),.Data showed that strawberry contains the highest amount of ash (2.78\%), followed by dry MA extract $(2.45 \%)$, whereas guava has the lowest value $(0.95 \%)$, these results go in agreement with those found by Al-Dabbas et al.,(2010). In addition, data indicates that the highest content of total carbohydrates $(7.29 \%)$ in guava, followed by strawberry $(7.27 \%)$, however the lowest content was in fresh MA extract (3.13\%), these results agreement with those found by Khattab and Shak, (2012).Table (3) registered that the highest fiber content was $2.90 \%$ in dry moringa, followed by fresh moringa $(2.51 \%)$, 
while the lowest content was $1.09 \%$ for cantaloupe, which agree with the finding of Ndong and Wade(2007) .On the other hand, the data illustrated in Table (3) showed that the highest protein content was observed in fresh MA extract $(4.1 \%)$, followed by dry MA extract $(2.85 \%)$, whereas cantaloupe had the lowest content $(1.95 \%)$ which agree with the finding of Seshadri and Nambiar (2003). Fat registered the highest content in fresh MA extract(1.72\%), also dry MA extract $(1.61 \%)$, the lowest content was $0.16 \%$ in cantaloupe, which agree with the finding of Busani et al., (2011). The highest value was $54.83 \mathrm{kcal}$ was in guava, followed by $46.61 \mathrm{kcal}$ in dry MA extract, the lowest value was $38.29 \mathrm{kcal}$ in cantaloupe.

Table (3): Chemical composition of fresh fruit juice strawberry, guava, pomegranate and cantaloupe, fresh and dry leaves of Moringa aqueous extract (W.W)

\begin{tabular}{|l|c|c|c|c|c|c|c|}
\hline $\begin{array}{l}\text { Camples } \\
\text { of blends }\end{array}$ & $\begin{array}{c}\text { Moisture } \\
\%\end{array}$ & $\begin{array}{c}\text { Ash } \\
\%\end{array}$ & $\begin{array}{c}\text { Total } \\
\text { carbohydrates } \\
\%\end{array}$ & $\begin{array}{c}\text { Fiber } \\
\%\end{array}$ & $\begin{array}{c}\text { Protein } \\
\%\end{array}$ & $\begin{array}{c}\text { Fat } \\
\%\end{array}$ & $\begin{array}{r}\text { Energy } \\
\text { (Kcal) }\end{array}$ \\
\hline Fresh MA & $87.04^{\mathrm{b}}$ & $1.50^{\mathrm{c}}$ & $3.13^{\mathrm{c}}$ & $2.51^{\mathrm{ab}}$ & $4.1^{\mathrm{a}}$ & $1.72^{\mathrm{a}}$ & $44.4^{\mathrm{b}}$ \\
extract & \pm 0.03 & \pm 0.00 & \pm 0.00 & \pm 0.01 & \pm 0.07 & \pm 0.01 & \pm 0.31 \\
\hline Dry MA & $85.01^{\mathrm{c}}$ & $2.45^{\mathrm{b}}$ & $5.18^{\mathrm{b}}$ & $2.90^{\mathrm{a}}$ & $2.85^{\mathrm{a}}$ & $1.61^{\mathrm{a}}$ & $46.61^{\mathrm{b}}$ \\
extract & \pm 0.0 & \pm 0.06 & \pm 0.21 & \pm 0.36 & \pm 0.20 & \pm 0.08 & \pm 0.30 \\
\hline Strawberry & $86.45^{\mathrm{b}}$ & $2.78^{\mathrm{a}}$ & $7.27^{\mathrm{a}}$ & $1.12^{\mathrm{c}}$ & $1.96^{\mathrm{c}}$ & $0.42^{\mathrm{c}}$ & $40.7^{\mathrm{e}}$ \\
& \pm 0.06 & \pm 0.31 & \pm 0.36 & \pm 0.54 & \pm 0.11 & \pm 0.42 & \pm 0.52 \\
\hline Guava & $86.5^{\mathrm{b}}$ & $0.95^{\mathrm{d}}$ & $7.29^{\mathrm{a}}$ & $1.28^{\mathrm{b}}$ & $2.03^{\mathrm{b}}$ & $1.95^{\mathrm{b}}$ & $54.84^{\mathrm{d}}$ \\
& \pm 0.21 & \pm 0.71 & \pm 0.13 & \pm 0.08 & \pm 0.50 & \pm 0.05 & \pm 0.18 \\
\hline Pomegranat & $87.91^{\mathrm{a}}$ & $1.12^{\mathrm{c}}$ & $7.49^{\mathrm{bc}}$ & $1.31^{\mathrm{b}}$ & $2.13^{\mathrm{b}}$ & $1.04^{\mathrm{e}}$ & $43.84^{\mathrm{e}}$ \\
e & \pm 0.53 & \pm 0.07 & \pm 0.26 & \pm 0.43 & \pm 0.33 & \pm 0.11 & \pm 0.52 \\
\hline Cantaloupe & $89.48^{\mathrm{a}}$ & $1.06^{\mathrm{b}}$ & $6.26^{\mathrm{a}}$ & $1.09^{\mathrm{c}}$ & $1.95^{\mathrm{c}}$ & $0.16^{\mathrm{d}}$ & $38.28^{\mathrm{ab}}$ \\
& \pm 0.42 & \pm 0.20 & \pm 0.46 & \pm 0.45 & \pm 0.00 & \pm 0.04 & \pm 0.21 \\
\hline
\end{tabular}

a,b,c Mean in the same row are bearing letters, differed significantly at $(\mathrm{P}<0.05)$.

$\mathrm{MA}=$ Moringa aqueous

\section{Physicochemical evaluation of juice, fresh and dry leaves of Moringa aqueous extract:}

The results given in Table (4) shows physical properties of fresh Moringa aqueous (MA) extract, dry MA extract and juices (strawberry, guava, pomegranate and cantaloupe).It is noticeable that there were significant differences between samples in total sugar, reducing sugar, nonreducing sugar, total soluble solids, total titraatable acidity, and $\mathrm{pH}$-value. Results showed that the highest content of total sugar was for pomegranate $(9.57 \%)$, followed by guava $(9.42 \%)$, while the lowest was in dry MA extract $(4.95 \%)$. Results showed in Table (4) revealed that the highest 
reducing sugar content was $7.54 \%$ for pomegranate, followed by strawberry $(5.08 \%)$, while the lowest was $3.77 \%$ for dry MA extract. Also, Nonreducing sugar represented the highest content $(4.54 \%)$ in guava, followed by $(2.03 \%)$ in pomegranate $(1.5 \%)$ in fresh MA extract $(1.4 \%)$ in strawberry, (1.18\%) dry MA extract, however, the lowest content was $0.96 \%$ in cantaloupe, which agree with the finding of Al-Kharusi, et al., (2009).Total soluble solids content was $12.10 \%$ for pomegranate as the highest content followed by $(9.01 \%)$ for cantaloupe, while the lowest content was $6.09 \%$ for fresh MA extract . Previous results relatively agree with the finding of Karol, et al., (2012). Total titraatable acidity represented in the same table indicated that the highest content was $2.9 \%$ for dry MA extract, followed by fresh MA extract (2.7\%), while cantaloupe had thelowest content (1.10\%).On the other hand the highest $\mathrm{pH}$ value was observed in dry MA extract (7.07\%), followed by fresh MA extract (6.03\%), while the lowest value was $5.30 \%$ for guava which agree with the finding of Alothman et al., (2009).

Table (4): Physicochemical evaluation of juice, fresh and dry leaves of MA extract.

\begin{tabular}{|l|c|c|c|c|c|c|}
\hline \multicolumn{1}{|c|}{ Content } & $\begin{array}{c}\text { Total } \\
\text { sugars } \\
\text { \% } \\
\text { of blends }\end{array}$ & $\begin{array}{c}\text { Reducing } \\
\text { Sugars } \\
\text { \% }\end{array}$ & $\begin{array}{c}\text { Non- } \\
\text { Reducing } \\
\text { Sugars } \\
\text { \% }\end{array}$ & $\begin{array}{c}\text { Total } \\
\text { soluble } \\
\text { Solids } \\
\%\end{array}$ & $\begin{array}{c}\text { Total } \\
\text { titratable } \\
\text { Acidity } \\
\%\end{array}$ & $\begin{array}{c}\text { pH } \\
\text { value }\end{array}$ \\
\hline Fresh MA & $6.19^{\mathrm{c}}$ & $4.69^{\mathrm{d}}$ & $1.5^{\mathrm{c}}$ & $6.09^{\mathrm{d}}$ & $2.7^{\mathrm{ab}}$ & $6.03^{\mathrm{b}}$ \\
extract & \pm 0.05 & \pm 0.31 & \pm 0.54 & \pm 0.20 & \pm 0.53 & \pm 0.06 \\
\hline Dry MA & $4.95^{\mathrm{d}}$ & $3.77^{\mathrm{e}}$ & $1.18^{\mathrm{cd}}$ & $7.43^{\mathrm{c}}$ & $2.9^{\mathrm{a}}$ & $7.07^{\mathrm{a}}$ \\
extract & \pm 2.30 & \pm 0.46 & \pm 0.23 & \pm 0.37 & \pm 0.50 & \pm 0.23 \\
\hline Strawberry & $7.2^{\mathrm{b}}$ & $5.80^{\mathrm{b}}$ & $1.4^{\mathrm{c}}$ & $7.5^{\mathrm{c}}$ & $1.52^{\mathrm{c}}$ & $5.51^{\mathrm{c}}$ \\
& \pm 0.60 & \pm 0.30 & \pm 0.16 & \pm 0.34 & \pm 0.21 & \pm 0.09 \\
\hline Guava & $9.42^{\mathrm{a}}$ & $5.08^{\mathrm{bc}}$ & $4.34^{\mathrm{a}}$ & $8.80^{\mathrm{b}}$ & $2.05^{\mathrm{b}}$ & $5.30^{\mathrm{c}}$ \\
& \pm 0.23 & \pm 0.03 & \pm 0.01 & \pm 0.02 & \pm 0.45 & \pm 0.32 \\
\hline Pomegranate & $95^{5 \mathrm{a}}$ & $7.54^{\mathrm{a}}$ & $2.03^{\mathrm{b}}$ & $12.10^{\mathrm{a}}$ & $2.82^{\mathrm{a}}$ & $6.03^{\mathrm{b}}$ \\
& \pm 0.10 & \pm 0.12 & \pm 0.10 & \pm 0.17 & \pm 0.32 & \pm 0.62 \\
\hline Cantaloupe & $5.42^{\mathrm{cd}}$ & $4.46^{\mathrm{d}}$ & $0.96^{\mathrm{e}}$ & $9.013^{\mathrm{b}}$ & $1.10^{\mathrm{cd}}$ & $6.04^{\mathrm{b}}$ \\
& \pm 0.20 & \pm 0.08 & \pm 0.04 & \pm 0.07 & \pm 0.74 & \pm 0.02 \\
\hline
\end{tabular}

a,b,c Mean in the same row are bearing letters , differed significantly at $(\mathrm{P}<0.05)$.

MA= Moringa aqueous 


\section{Total carotenoids (as $\beta$-Carotene) ascorbic acid and phenolic compound of raw juice, fresh and dry leaves of Moringa aqueous extract}

It is noticeable from Table (5) that there were significant differences between fresh fruits and Moringa aqueous (MA) extract content in total carotenoids (as $\beta$-Carotene), vitamins and phenolic compounds $(\mathrm{mg} / 100 \mathrm{~g})$. Results in Table (5) indicates thattotalcarotenoids (as $\beta$-Carotene) content represented the highest content in dry MA extract $(27.42 \mathrm{mg} / 100 \mathrm{~g})$, followed by fresh MA extract $(24.36 \mathrm{mg} / 100 \mathrm{~g})$, while pomegranate represented the lowest value $(6.02 \mathrm{mg} / 100 \mathrm{~g})$. These results agree with the finding of Mariäa, et al.,(2002). Vitamin E- Tocopherols Acetate recorded the highest value $(23 \mathrm{mg} / 100 \mathrm{~g})$ in dry MA extract, followed by fresh MA extract $(20.5 \mathrm{mg} / 100 \mathrm{~g})$, while the lowest value was $0.1 \mathrm{mg} / 100 \mathrm{~g}$ for cantaloupe, vitamin B1 - Thiamin showed that the highest value was in dry MA extract $(2.6 \mathrm{mg} / 100 \mathrm{~g})$, followed by fresh MA extract $(0.21 \mathrm{mg} / 100 \mathrm{~g})$, while the lowest value was for pomegranate $(0.024 \mathrm{mg} / 100 \mathrm{~g})$. Vitamin B2 Riboflavincontent represent the highest content in dry MA extract $(30.5 \mathrm{mg} / 100 \mathrm{~g})$, followed by fresh MA extract $(18.05 \mathrm{mg} / 100 \mathrm{~g})$, while the lowest contained was $2.02 \mathrm{mg} / 100 \mathrm{~g}$ for cantaloupe. Vitamin B3 - Nicotinic Acidrecorded that the highest value was $8.2 \mathrm{mg} / 100 \mathrm{~g}$, for dry MA extract, followed by $(3.8 \mathrm{mg} / 100 \mathrm{~g})$ for fresh MA extract, while the lowest was $1.29 \mathrm{mg} / 100 \mathrm{~g}$ for pomegranate. On the other hand, data illustrated in Table (5) showed that the highest vitamin $\mathrm{C}$ content was observed in dry MA extract $(52.3 \mathrm{mg} / 100 \mathrm{~g})$, followed by fresh MA extract $(51.8 \mathrm{mg} / 100 \mathrm{~g})$, whereas pomegranate had the lowest content $(16.2 \mathrm{mg} / 100 \mathrm{~g})$, phenolic compounds showed that the highest value in dry MA extract $(41.56 \mathrm{~g} / 100$ $\mathrm{mg})$, followed by fresh MA extract $(22.38 \mathrm{~g} / 100 \mathrm{mg})$, cantaloupe recorded the lowest value (19.5g/100g), These results agree with Arun et al., (2013).

Mineral content of fresh juice, strawberry, guava, pomegranate, cantaloupe, fresh and dry leaves of moringa aqueous extract (mg/100g) W.W

Calcium, potassium, magnesium, sodium, iron, zinc, and manganese are presented in Table 6. Data showed that there were significant differences $(\mathrm{P}<0.05)$ in mineral content, in calcium content as the highest was $48 \mathrm{mg}$ in dry MA extract, followed by fresh MA extract (44mg), , while the lowest was $9.09 \mathrm{mg}$ in cantaloupe which agree with the finding of Broin, (2006). Magnesium represented the highest content was $98.04 \mathrm{mg}$ for dry MA extract, followed by $(51 \mathrm{mg})$ for fresh MA extract, while the lowest was $12 \mathrm{mg}$ for pomegranate. These results agree with the finding of Nkafamiya, et al., (2010). Data in the same Table showed that the highest sodium content was observed highest in dry MA extract (10.24mg), followed by 
Table (5): Total carotenoids (as $\beta$-Carotene) ascorbic acid and phenolic compound of freshjuice, fresh and dry leaves of moringa aqueous extract $(\mathrm{mg} / 100 \mathrm{~g}) \mathrm{W} . \mathrm{W}$.

\begin{tabular}{|c|c|c|c|c|c|c|c|}
\hline \multirow[t]{2}{*}{ Content } & \multirow[b]{2}{*}{$\begin{array}{c}\text { Total } \\
\text { carotenoids } \\
\text { (as } \\
\beta \text {-Carotene) } \\
\text { (mg/100g }\end{array}$} & \multicolumn{5}{|c|}{ Vitamins $-(\mathrm{mg} / \mathbf{1 0 0 g})$} & \multirow{2}{*}{$\begin{array}{l}\text { *Phenolic } \\
\text { compounds } \\
\text { (mg/100g) }\end{array}$} \\
\hline & & $\begin{array}{l}\text { Vitamin E- } \\
\text { Tocopherols } \\
\text { Acetate }(\mathrm{mg})\end{array}$ & $\begin{array}{c}\text { Vitamin } \\
\text { B1 - } \\
\text { Thiamin } \\
\text { (mg) }\end{array}$ & $\begin{array}{c}\text { Vitamin } \\
\text { B2- } \\
\text { Riboflavin }\end{array}$ & $\begin{array}{c}\text { Vitamin } \\
\text { B3- } \\
\text { Nicotini } \\
\text { c Acid } \\
\text { (mg) }\end{array}$ & $\begin{array}{c}\text { Vitamin } \\
\text { C- } \\
\text { Ascorbic } \\
\text { Acid } \\
\text { (mg) }\end{array}$ & \\
\hline $\begin{array}{l}\text { Fresh MA } \\
\text { extract }\end{array}$ & $\begin{array}{l}24.36^{6} \\
\pm 0.08\end{array}$ & $\begin{array}{c}23^{\mathrm{a}} \\
\pm 0.60\end{array}$ & $\begin{array}{l}4.21^{b} \\
\pm 0.43\end{array}$ & $\begin{array}{l}18.05^{b} \\
\pm 0.60\end{array}$ & $\begin{array}{c}4.8^{\mathrm{b}} \\
\pm 0.09\end{array}$ & $\begin{array}{c}51.84^{\mathrm{a}} \\
\pm 0.0\end{array}$ & $\begin{array}{l}22.38^{\mathrm{a}} \\
\pm 0.08\end{array}$ \\
\hline $\begin{array}{l}\text { Dry MA } \\
\text { extract }\end{array}$ & $\begin{array}{l}27.42^{\mathrm{a}} \\
\pm 0.54\end{array}$ & $\begin{array}{l}25.5^{\mathrm{a}} \\
\pm 0.21\end{array}$ & $\begin{array}{c}6.6^{\mathrm{a}} \\
\pm 0.42\end{array}$ & $\begin{array}{l}30.5^{\mathrm{a}} \\
\pm 0.00\end{array}$ & $\begin{array}{c}8.2^{\mathrm{a}} \\
\pm 0.21\end{array}$ & $\begin{array}{l}52.3^{\mathrm{a}} \\
\pm 0.16\end{array}$ & $\begin{array}{l}21.56^{\mathrm{b}} \\
\pm 0.60\end{array}$ \\
\hline Strawberry & $\begin{array}{l}20.08^{\mathrm{c}} \\
\pm 0.32\end{array}$ & $\begin{array}{l}0.29^{\mathrm{e}} \\
\pm 0.07\end{array}$ & $\begin{array}{l}1.02^{\mathrm{e}} \\
\pm 0.16\end{array}$ & $\begin{array}{l}4.02^{\mathrm{dc}} \\
\pm 0.17\end{array}$ & $\begin{array}{l}0.36^{\mathrm{e}} \\
\pm 0.43\end{array}$ & $\begin{array}{l}18.8^{\mathrm{bc}} \\
\pm 0.10\end{array}$ & $\begin{array}{l}20.2^{\mathrm{c}} \\
\pm 0.34\end{array}$ \\
\hline Guava & $\begin{array}{l}21.74^{\mathrm{c}} \\
\pm 0.12\end{array}$ & $\begin{array}{l}0.73^{\mathrm{c}} \\
\pm 0.11\end{array}$ & $\begin{array}{l}2.11^{\mathrm{c}} \\
\pm 0.06\end{array}$ & $\begin{array}{l}7.04^{\mathrm{c}} \\
\pm 0.16\end{array}$ & $\begin{array}{l}1.08^{\mathrm{bc}} \\
\pm 0.31\end{array}$ & $\begin{array}{l}22.8^{\mathrm{b}} \\
\pm 0.34\end{array}$ & $\begin{array}{l}22.60^{\mathrm{a}} \\
\pm 0.16\end{array}$ \\
\hline Pomegranate & $\begin{array}{l}6.02^{\mathrm{d}} \\
\pm 0.16\end{array}$ & $\begin{array}{l}0.60^{\mathrm{d}} \\
\pm 0.34\end{array}$ & $\begin{array}{l}1.06^{\mathrm{d}} \\
\pm 0.36\end{array}$ & $\begin{array}{l}9.05^{\mathrm{b}} \\
\pm 0.13\end{array}$ & $\begin{array}{c}0.29^{f} \\
\pm 0.16\end{array}$ & $\begin{array}{l}16.2^{\mathrm{e}} \\
\pm 0.30\end{array}$ & $\begin{array}{l}21.2^{\mathrm{b}} \\
\pm 0.13\end{array}$ \\
\hline Cantaloupe & $\begin{array}{l}20.20^{\mathrm{c}} \\
\pm 0.10\end{array}$ & $\begin{array}{c}0.1^{\mathrm{c}} \\
\pm 0.11 \\
\end{array}$ & $\begin{array}{c}0.1^{\mathrm{c}} \\
\pm 0.16 \\
\end{array}$ & $\begin{array}{l}2.02^{\mathrm{c}} \\
\pm 0.01\end{array}$ & $\begin{array}{c}1.3^{\mathrm{c}} \\
\pm 0.07\end{array}$ & $\begin{array}{l}36.0^{\mathrm{b}} \\
\pm 0.16 \\
\end{array}$ & $\begin{array}{l}19.5^{\mathrm{c}} \\
\pm 0.10 \\
\end{array}$ \\
\hline
\end{tabular}

* Total phenolic compounds were measured as catechol $\mathrm{MA}=$ Moringa aqueou

a, b, c Mean in the same row are bearing letters, differed significantly at $(\mathrm{P}<0.05)$.

Table (6): Mineral content of fresh juice, strawberry, guava, pomegranate, cantaloupe, fresh and dry moringa leaves aqueous extract $(\mathrm{mg} / 100 \mathrm{~g}) \mathrm{W}$

\begin{tabular}{|l|c|c|c|c|c|c|c|}
\hline \multicolumn{1}{|c|}{ Content } & $\begin{array}{c}\text { Ca } \\
(\mathbf{m g})\end{array}$ & $\begin{array}{c}\mathbf{K} \\
(\mathbf{m g})\end{array}$ & $\begin{array}{c}\mathbf{M g} \\
(\mathbf{m g})\end{array}$ & $\begin{array}{c}\mathbf{N a} \\
(\mathbf{m g})\end{array}$ & $\begin{array}{c}\mathbf{F e} \\
(\mathbf{m g})\end{array}$ & $\begin{array}{c}\mathbf{Z n} \\
(\mathbf{m g})\end{array}$ & $\begin{array}{c}\text { Mn } \\
(\mathbf{m g})\end{array}$ \\
\hline Flends of & & & & & & & \\
\hline Fresh MA extract & $44.0^{\mathrm{b}}$ & $41.0^{\mathrm{a}}$ & $51.0^{\mathrm{b}}$ & $90^{\mathrm{a}}$ & $10.8^{\mathrm{a}}$ & $0.6^{\mathrm{b}}$ & $25.0^{\mathrm{b}}$ \\
& \pm 0.03 & \pm 0.12 & \pm 0.14 & \pm 0.12 & \pm 0.01 & \pm 0.34 & \pm 0.12 \\
\hline Dry MA & $48.23^{\mathrm{a}}$ & $80.63^{\mathrm{a}}$ & $98.04^{\mathrm{a}}$ & $10.24^{\mathrm{b}}$ & $14.45^{\mathrm{b}}$ & $1.63^{\mathrm{a}}$ & $27.21^{\mathrm{a}}$ \\
extract & \pm 0.4 & \pm 0.0 & \pm 0.34 & \pm 0.21 & \pm 0.34 & \pm 0.34 & \pm 0.34 \\
\hline Strawberry & $16.04^{\mathrm{d}}$ & $15.56^{\mathrm{b}}$ & $13.72^{\mathrm{d}}$ & $1.44^{\mathrm{e}}$ & $2.59^{\mathrm{d}}$ & $0.20^{\mathrm{d}}$ & $2.56^{\mathrm{c}}$ \\
& \pm 0.34 & \pm 0.30 & \pm 0.31 & \pm 0.11 & \pm 0.22 & \pm 0.13 & \pm 0.31 \\
\hline Guava & $17.0^{\mathrm{d}}$ & $17.0^{\mathrm{a}}$ & $22.10^{\mathrm{c}}$ & $2.50^{\mathrm{ed}}$ & $3.70^{\mathrm{c}}$ & $0.94^{\mathrm{e}}$ & $1.12^{\mathrm{d}}$ \\
& \pm 0.42 & \pm 0.54 & \pm 0.24 & \pm 0.10 & \pm 0.36 & \pm 0.02 & \pm 0.04 \\
\hline Pomegranate & $20.0^{\mathrm{c}}$ & $19.0^{\mathrm{b}}$ & $12^{\mathrm{d}}$ & $3.0^{\mathrm{c}}$ & $1.30^{\mathrm{e}}$ & $0.35^{\mathrm{c}}$ & $1.19^{\mathrm{e}}$ \\
& \pm 0.12 & \pm 0.12 & \pm 0.33 & \pm 0.12 & \pm 0.15 & \pm 0.04 & \pm 0.06 \\
\hline Cantaloupe & $9.09^{\mathrm{e}}$ & $17.0^{\mathrm{c}}$ & $12.2^{\mathrm{d}}$ & $11.3^{\mathrm{a}}$ & $2.21^{\mathrm{f}}$ & $0.30^{\mathrm{c}}$ & $1.1^{\mathrm{e}}$ \\
& \pm 0.42 & \pm 0.42 & \pm 0.34 & \pm 0.03 & \pm 0.01 & \pm 0.14 & \pm 0.05 \\
\hline
\end{tabular}

a, b, c Mean in the same row are bearing letters, differed significantly at $(\mathrm{P}<0.05)$.

MA: Moringa aqueous 
fresh MA extract (9mg), whereas the lowest content was $1.44 \mathrm{mg}$ in strawberry. Iron represented the highest content was 14.54 for dry MA extract, followed by (10.8) for fresh MA extract, while the lowest content was $0.21 \mathrm{mg}$ for cantaloupe .These results agree with Mogobe et al., (2015). Zink showed that the highest content was $1.63 \mathrm{mg}$ in dry MA extract, followed by $(0.6 \mathrm{mg})$ in fresh MA extract. The lowest content was $0.140 \mathrm{mg}$ for guava, Manganese showed that the highest value was $27.21 \mathrm{mg}$ in dry MA extract, followed by $(25 \mathrm{mg})$ in fresh MA extract, the lowest content was $1.1 \mathrm{mg}$ in cantaloupe.

\section{Chemical composition of fresh juice with moringa mixes:}

Moisture, ash, total sugars, reducing sugars, non- reducing sugars, total soluble solids, total titratable acidity and $\mathrm{pH}$ value are presented in Table 7. Data show that there was significant differences $(\mathrm{P}<0.05)$ in chemical composition of fresh juice blends. Moisture revealed that the highest content was 50\% pomegranate juice $+50 \%$ MA extract93.34\% followed by $50 \%$ cantaloupe juice $+50 \%$ MA extract $(92.05 \%)$, while the lowest content was $85.01 \%$ MA extract This finding agrees with those obtained by Shahnawaz et al., (2013).Ash showed that the highest content was $50 \%$ Strawberry juice+ $50 \%$ MA extract(3.05\%), followed by MA extract $(2.45 \%)$, while the lowest content was $0.67 \%$ in $50 \%$ guava juice+ $50 \%$ MA extract. These results are in agreement with Hemalatha and Anbuselvi (2013).

Total sugars revealed that the highest content was $8.02 \%$ in $50 \%$ pomegranate juice $+50 \%$ MA extract, followed by $50 \%$ guava juice $+50 \%$ MA extract(7.91\%), while the lowest content was $4.95 \%$ in MA extract. These agreement agree with Akther et al. (2012).

Reducing Sugars, showed that the highest content was $6.82 \%$ in $50 \%$ pomegranate juice $+50 \%$ MA extract $(7.82 \%)$, followed by $50 \%$ guava juice $+50 \%$ MA extract(5.71\%), while the lowest content was $3.77 \%$ in MA extract. Non- Reducing Sugars, represented in the same table the highest value was $2.20 \%$ in $50 \%$ guava juice $+50 \%$ MA extract, followed by $50 \%$ pomegranate juice+ $50 \%$ MA extract $(1.2 \%$ ), while the lowest was $0.96 \%$ in $50 \%$ cantaloupe juice $+50 \%$ MA extract.

Total soluble solids revealed that the highest content was $50 \%$ pomegranate juice $+50 \%$ MA extract $(9.43 \%)$ followed by $50 \%$ cantaloupe juice $+50 \%$ MA extract $(7.98 \%$ ), while the lowest value was $50 \%$ Strawberry juice $+50 \%$ MA extract $(4,25 \%)$, which agree with the finding of El-sherif and Alghamdi, (2013). 
Table (7): Chemical composition of fresh juice with moringa mixes

\begin{tabular}{|c|c|c|c|c|c|c|c|c|}
\hline Content. & $\begin{array}{c}\text { Moisture } \\
\%\end{array}$ & $\begin{array}{c}\text { Ash } \\
\%\end{array}$ & $\begin{array}{l}\text { Total } \\
\text { sugars } \\
\%\end{array}$ & \begin{tabular}{|} 
Reducin \\
g \\
Sugars \\
$\%$
\end{tabular} & $\begin{array}{l}\text { Non- } \\
\text { Reducing } \\
\text { Sugars } \\
\quad \%\end{array}$ & $\begin{array}{c}\text { Total } \\
\text { soluble } \\
\text { solids } \\
\%\end{array}$ & $\begin{array}{c}\text { Total } \\
\text { titratable } \\
\text { Acidity } \\
\%\end{array}$ & $\begin{array}{l}\mathrm{pH}- \\
\text { value }\end{array}$ \\
\hline MA extract & $\begin{array}{c}85.01^{\mathrm{d}} \\
\pm 0.0\end{array}$ & $\begin{array}{l}2.45^{\mathrm{a}} \\
\pm 0.0\end{array}$ & $\begin{array}{l}4.95^{\mathrm{cd}} \\
\pm 2.3\end{array}$ & $\begin{array}{l}3.77^{\mathrm{d}} \\
\pm 0.46\end{array}$ & $\begin{array}{r}1.18^{b} \\
\pm 0.23\end{array}$ & $\begin{array}{l}7.43^{\mathrm{a}} \\
\pm 0.3\end{array}$ & $\begin{array}{l}2.9^{\mathrm{a}} \pm \\
0.50\end{array}$ & $\begin{array}{r}7.07^{\mathrm{a}} \\
\pm 0.23\end{array}$ \\
\hline $\begin{array}{l}50 \% \text { Strawberry } \\
\text { juice+ 50\% MA } \\
\text { Extract }\end{array}$ & $\begin{array}{c}91.95^{\mathrm{ab}} \\
\pm 0.6\end{array}$ & $\begin{array}{l}2.05^{\mathrm{b}} \\
\pm 0.2\end{array}$ & $\begin{array}{l}5.28^{\mathrm{e}} \\
\pm 0.6\end{array}$ & $\begin{array}{l}4.20^{\mathrm{c}} \\
\pm 0.42\end{array}$ & $\begin{array}{l}1.08^{\mathrm{c}} \\
\pm 0.61\end{array}$ & $\begin{array}{l}4.25^{\mathrm{d}} \\
\pm 0.5\end{array}$ & $\begin{array}{l}1.20^{\mathrm{cd}} \\
\pm 0.4\end{array}$ & $\begin{array}{l}6.32^{b} \\
\pm 0.30\end{array}$ \\
\hline $\begin{array}{l}\mathbf{5 0 \%} \text { Guava juice+ } \\
\mathbf{5 0 \%} \text { MA Extract }\end{array}$ & $\begin{array}{c}88.90^{\mathrm{c}} \\
\pm 0.5\end{array}$ & $\begin{array}{l}0.67^{\mathrm{d}} \\
\pm 0.2\end{array}$ & $\begin{array}{l}7.91^{b} \\
\pm 0.2\end{array}$ & $\begin{array}{l}5.71^{b} \\
\pm 0.3\end{array}$ & $\begin{array}{l}2.20^{\mathrm{a}} \\
\pm 0.54\end{array}$ & $\begin{array}{l}4.56^{\mathrm{d}} \\
\pm 0.2\end{array}$ & $\begin{array}{l}1.8^{\mathrm{c}} \\
\pm 0.0\end{array}$ & $\begin{array}{l}6.62^{b} \\
\pm 0.20\end{array}$ \\
\hline $\begin{array}{c}50 \% \text { Pomegranate+ } \\
50 \% \text { MA Extract }\end{array}$ & $\begin{array}{c}93.43^{\mathrm{a}} \\
\pm 0.6\end{array}$ & $\begin{array}{l}1.93^{\mathrm{c}} \\
\pm 0.0\end{array}$ & $\begin{array}{l}8.02^{\mathrm{a}} \\
\pm 0.3\end{array}$ & $\begin{array}{r}6.82^{\mathrm{a}} \\
\pm 0.52\end{array}$ & $\begin{array}{c}1.2^{\mathrm{b}} \\
\pm 0.40\end{array}$ & $\begin{array}{l}6.34^{\mathrm{b}} \\
\pm 0.21\end{array}$ & $\begin{array}{l}2.2^{b} \\
\pm 0.6\end{array}$ & $\begin{array}{l}6.78^{\mathrm{b}} \\
\pm 0.04\end{array}$ \\
\hline $\begin{array}{l}\text { 50\% Cantaloupe+ } \\
\text { 50\% MA Extract }\end{array}$ & $\begin{array}{c}92.05^{\mathrm{a}} \\
\pm 0.2\end{array}$ & $\begin{array}{l}1.89^{c} \\
\pm 0.4\end{array}$ & $\begin{array}{l}5.22^{\mathrm{c}} \\
\pm 0.4\end{array}$ & $\begin{array}{l}4.26^{\mathrm{c}} \\
\pm 0.04\end{array}$ & $\begin{array}{l}0.96^{\mathrm{d}} \\
\pm 0.20\end{array}$ & $\begin{array}{l}5.98^{\mathrm{c}} \\
\pm 0.2\end{array}$ & $\begin{array}{l}0.98^{\mathrm{e}} \\
\pm 0.3\end{array}$ & $\begin{array}{l}6.90^{\mathrm{ab}} \\
\pm 0.12\end{array}$ \\
\hline
\end{tabular}

a, b, c Mean in the same row are bearing letters, differed significantly at $(\mathrm{P}<0.05)$.

MA: Moringa aqueous

Total titratable acidity represented that the highest content was $2.9 \%$ in MA extract, followed by $50 \%$ pomegranate juice $+50 \%$ MA extract, $(2.02 \%)$, while the lowest content was in $50 \%$ cantaloupe juice+ $50 \%$ MA extract $(0.98 \%) \mathrm{pH}$ value revealed that the highest content was $7.07 \%$ in MA extract, followed by $50 \%$ cantaloupe juice $+50 \%$ MA extract $(6.90 \%)$, while the lowest content was $50 \%$ strawberry juice+ 50\% MA extract(6.32\%). These results agree with the findings of Leghari et al., (2013).

\section{Minerals content of fresh juice with moringa mixes}

Calcium, potassium, magnesium, sodium, iron, zinc and magnesium are presented in Table 8. Data showed that there were significant differences $(\mathrm{P}<0.05)$ in minerals content of fresh juices blends in calcium content as the highest was in MA extract $(48.23 \mathrm{mg} / 100 \mathrm{~g})$, followedby $50 \%$ strawberry juice $+50 \%$ MA extract $(30.21 \mathrm{mg} / 100 \mathrm{~g})$, while the lowest was 22.65 $\mathrm{mg} / 100 \mathrm{~g}$ in $50 \%$ cantaloupe+ 50\% MA extract these finding agree with those found by Awsi, and Masih, (2012). In Potassium, the highest content was $50 \%$ pomegranate+ $50 \%$ MA extract $(82.01 \mathrm{mg} / 100 \mathrm{~g})$, followed by MA extract $(80.62 \mathrm{mg} / 100 \mathrm{~g})$, while the lowest was $50 \%$ guava juice+ $50 \%$ MA extract $(37.54 \mathrm{mg} / 100 \mathrm{~g})$. On the other hand, the highest Magnesium content was observed in MA extract $(98.04 \mathrm{mg} / 100 \mathrm{~g})$, followed by $50 \%$ strawberry juice $+50 \%$ MA extract $(63.72 \mathrm{mg} / 100 \mathrm{~g})$, while the lowest was $33.12 \mathrm{mg} / 100 \mathrm{~g}$ in the lowest was $33.12 \mathrm{mg} / 100 \mathrm{~g}$ in $50 \%$ pomegranate $+50 \%$ 
Table (8): Minerals content of fresh juice with moringa mixes

\begin{tabular}{|c|c|c|c|c|c|c|c|}
\hline $\begin{array}{l}\text { Content. } \\
\text { Sample of }\end{array}$ & $\begin{array}{c}\mathrm{Ca} \\
(\mathrm{mg} / \\
100 \mathrm{~g})\end{array}$ & $\begin{array}{c}\mathrm{K} \\
(\mathbf{m g} / \\
\mathbf{1 0 0 g})\end{array}$ & $\begin{array}{c}\text { Mg } \\
(\mathbf{m g} / \\
100 \mathrm{~g})\end{array}$ & $\begin{array}{l}\mathrm{Na} \\
(\mathrm{mg} / \\
100 \mathrm{~g})\end{array}$ & $\begin{array}{l}\mathrm{Fe} \\
(\mathrm{mg} / \\
100 \mathrm{~g})\end{array}$ & $\begin{array}{l}\mathrm{Zn} \\
(\mathbf{m g} / \\
\mathbf{1 0 0 g})\end{array}$ & $\begin{array}{l}\text { Mn } \\
(\mathrm{mg} / \\
100 \mathrm{~g})\end{array}$ \\
\hline $\mathbf{M}$ & $\begin{array}{l}48.23^{\mathrm{a}} \\
\pm 0.01\end{array}$ & $\begin{array}{c}80.62^{\mathrm{ab}} \\
\pm 0.54\end{array}$ & $\begin{array}{l}98.04^{\mathrm{a}} \\
\pm 0.70\end{array}$ & $\begin{array}{l}10.24^{\mathrm{c}} \\
\pm 0.68\end{array}$ & $\begin{array}{l}14.54^{\mathrm{a}} \\
\pm 0.74\end{array}$ & $\begin{array}{l}1.63^{\mathrm{d}} \\
\pm 0.44\end{array}$ & $\begin{array}{l}27.21^{\mathrm{e}} \\
\pm 0.12\end{array}$ \\
\hline $\begin{array}{l}\mathbf{5 0 \%} \text { Strawberry + } \\
\mathbf{5 0 \%} \text { MA extract }\end{array}$ & $\begin{array}{l}30.21^{\mathrm{b}} \\
\pm 0.64\end{array}$ & $\begin{array}{c}43.07^{\mathrm{c}} \\
\pm 0.34\end{array}$ & $\begin{array}{r}63.72^{\mathrm{b}} \\
\pm 030\end{array}$ & $\begin{array}{l}11.05^{\mathrm{b}} \\
\pm 0.20\end{array}$ & $\begin{array}{l}13.95^{\mathrm{b}} \\
\pm 0.66\end{array}$ & $\begin{array}{l}2.56^{\mathrm{c}} \\
\pm 0.01\end{array}$ & $\begin{array}{l}22.03^{\mathrm{a}} \\
\pm 0.08\end{array}$ \\
\hline $\begin{array}{l}\text { 50\% Guava juice + } \\
\text { 50\% MA extract }\end{array}$ & $\begin{array}{l}29.05^{\mathrm{b}} \\
\pm 0.55\end{array}$ & $\begin{array}{c}37.54^{\mathrm{d}} \\
\pm 0.04\end{array}$ & $\begin{array}{l}54.01^{\mathrm{c}} \\
\pm 0.14\end{array}$ & $\begin{array}{l}13.70^{\mathrm{a}} \\
\pm 0.93\end{array}$ & $\begin{array}{l}9.45^{\mathrm{c}} \\
\pm 0.62\end{array}$ & $\begin{array}{c}3.41^{\mathrm{b}} \\
\pm 0.32\end{array}$ & $\begin{array}{l}14.99^{b} \\
\pm 0.09\end{array}$ \\
\hline $\begin{array}{l}\mathbf{5 0 \%} \text { pomegranate + } \\
\mathbf{5 0 \%} \text { MA extract }\end{array}$ & $\begin{array}{l}25.51^{\mathrm{c}} \\
\pm 0.60\end{array}$ & $\begin{array}{l}82.01^{\mathrm{a}} \\
\pm 0.94\end{array}$ & $\begin{array}{l}33.12^{\mathrm{d}} \\
\pm 0.69\end{array}$ & $\begin{array}{l}11.12^{\mathrm{b}} \\
\pm 0.45\end{array}$ & $\begin{array}{l}8.61^{\mathrm{c}} \\
\pm 077\end{array}$ & $\begin{array}{l}6.35^{\mathrm{a}} \\
\pm 0.04\end{array}$ & $\begin{array}{l}13.86^{\mathrm{c}} \\
\pm 0.03\end{array}$ \\
\hline $\begin{array}{l}\mathbf{5 0 \%} \text { cantaloupe+ } \\
\mathbf{5 0 \%} \text { MA extract }\end{array}$ & $\begin{array}{l}22.65^{\mathrm{d}} \\
\pm 0.34\end{array}$ & $\begin{array}{l}81.06^{\mathrm{a}} \\
\pm 0.60\end{array}$ & $\begin{array}{c}34.03^{\mathrm{d}} \\
\pm 0.54\end{array}$ & $\begin{array}{l}12^{56 \mathrm{ab}} \\
\pm 0.24\end{array}$ & $\begin{array}{l}8.54^{\mathrm{c}} \\
\pm 0.11\end{array}$ & $\begin{array}{c}2.31^{\mathrm{c}} \\
\pm 0.14\end{array}$ & $\begin{array}{l}11.44^{\mathrm{d}} \\
\pm 0.674\end{array}$ \\
\hline
\end{tabular}

a, b, c Mean in the same row are bearing letters, differed significantly at $(\mathrm{P}<0.05)$.

MA: Moringa aqueous.

MA extract, these finding agree with chuku and chinaka, (2014).

In sodium, the highest content was $13.70 \mathrm{mg} / 100 \mathrm{~g}$ in $50 \%$ guava juice $+50 \%$ MA extract, followed by $50 \%$ cantaloupe+ $50 \%$ MA extract $(12.56 \mathrm{mg} / 100 \mathrm{~g})$, while the lowest value was $10.24 \mathrm{mg} / \mathrm{gin}$ moringa drink. The highest content for iron was $14.54 \mathrm{mg} / 100 \mathrm{~g}$ in MA extract, followed by $50 \%$ cantaloupe $+50 \%$ MA extract $(13.95 \mathrm{mg} / 100 \mathrm{~g})$, while the lowest content was $8.54 \mathrm{mg} / 100 \mathrm{~g}$ in $50 \%$ cantaloupe $+50 \%$ MA extract, In Zinc, the highest content was $6.35 \mathrm{mg} / 100 \mathrm{~g}$ in $50 \%$ pomegranate $+50 \%$ moringa drink, followed by $50 \%$ guava juice $+50 \%$ MA extract $(3.41 \mathrm{mg} / 100 \mathrm{~g})$. while the lowest value was $1.63 \mathrm{mg} / 100 \mathrm{~g}$ in MA extract, these finding agree with Ogunbanwo et al.,(2013) On the other hand, thehighest Magnesium content was observed in MA extract $(27.21 \mathrm{mg}$ $/ 100 \mathrm{~g})$, followed by $50 \%$ strawberry juice $+50 \%$ MA extract $(22.03 \mathrm{mg}$ $/ 100 \mathrm{~g}$ ), while the lowest was 11.44 in $50 \%$ cantaloupe juice $+50 \%$ MA extract. These finding agreed with Singh and Bunkar (2005).

Natural antioxidants and its activities of moringa juice mixes and moringa aqueous extract

Total polyphenols, total flavonoids, ascorbic acid, $\beta$-carotene, carotenoids and total antioxidant activity are presented in Table 9. Data 
Table (9): Natural antioxidants and its activities of moringa juice mixes and Moringa aqueous extract

\begin{tabular}{|c|c|c|c|c|c|c|}
\hline $\begin{array}{l}\text { Content } \\
\text { Sample of } \\
\text { blends }\end{array}$ & $\begin{array}{c}\text { Total } \\
\text { polyphenols } \\
(\mathrm{mg} / 100 \mathrm{~g})\end{array}$ & $\begin{array}{c}\text { Total } \\
\text { flavonoids } \\
(\mathrm{mg} / 100 \mathrm{~g})\end{array}$ & $\begin{array}{l}\text { Ascorbic } \\
\text { acid } \\
(\mathrm{mg} / 100 \mathrm{~g})\end{array}$ & $\begin{array}{c}\boldsymbol{\beta}- \\
\text { carotene } \\
(\mathrm{mg} / 100 \mathrm{~g})\end{array}$ & \begin{tabular}{|} 
Carotenoids \\
(mg/100 \\
g)
\end{tabular} & $\begin{array}{c}\text { Total } \\
\text { antioxidan } \\
\text { activity } \\
(\%)\end{array}$ \\
\hline MA extract & $\begin{array}{l}21.56^{\mathrm{d}} \\
+0.87 \\
\end{array}$ & $\begin{array}{c}17.98^{\mathrm{a}} \pm \\
0.79\end{array}$ & $\begin{array}{r}17.84^{\mathrm{a}} \\
+0.72 \\
\end{array}$ & $\begin{array}{r}13.59^{c} \\
+0.70\end{array}$ & $\begin{array}{r}27.42^{\mathrm{a}} \\
+0.67\end{array}$ & $\begin{array}{l}98.39^{\mathrm{a}} \\
+0.97\end{array}$ \\
\hline $\begin{array}{l}50 \% \text { Strawberry } \\
+\mathbf{5 0 \%} \text { MA extract }\end{array}$ & $\begin{array}{l}26.21^{\mathrm{b}} \\
\pm 0.32\end{array}$ & $\begin{array}{l}14.65^{\mathrm{c}} \\
\pm 0.23\end{array}$ & $\begin{array}{l}14.18^{\mathrm{c}} \\
\pm 0.30\end{array}$ & $\begin{array}{l}14.41^{\mathrm{b}} \\
\pm 0.2\end{array}$ & $\begin{array}{l}24.12^{\mathrm{bc}} \\
\pm 0.32\end{array}$ & $\begin{array}{c}93.57^{\mathrm{b}} \\
\pm 0.2\end{array}$ \\
\hline $\begin{array}{l}\text { 50\% Guava juice } \\
+\mathbf{5 0 \%} \text { MA extract }\end{array}$ & $\begin{array}{l}27.04^{\mathrm{a}} \\
\pm 0.73\end{array}$ & $\begin{array}{l}15.12^{\mathrm{b}} \\
+0.67\end{array}$ & $\begin{array}{l}16.02^{\mathrm{b}} \\
\pm 0.01\end{array}$ & $\begin{array}{l}15.65^{\mathrm{a}} \\
\pm 0.32\end{array}$ & $\begin{array}{l}25.43^{\mathrm{b}} \\
\pm 0.73\end{array}$ & $\begin{array}{l}99.26^{\mathrm{a}} \\
\pm 0.73\end{array}$ \\
\hline $\begin{array}{l}50 \% \text { pomegranate } \\
+50 \% \text { MA extract }\end{array}$ & $\begin{array}{l}26.56^{\mathrm{b}} \\
\pm 0.61\end{array}$ & $\begin{array}{l}14.43^{\mathrm{c}} \\
\pm 0.2\end{array}$ & $\begin{array}{l}13.34^{\mathrm{d}} \\
\pm 0.57\end{array}$ & $\begin{array}{l}14.31^{\mathrm{b}} \\
\pm 0.60\end{array}$ & $\begin{array}{l}19.43^{\mathrm{d}} \\
\pm 0.32\end{array}$ & $\begin{array}{l}88.07^{\mathrm{c}} \\
\pm 0.01\end{array}$ \\
\hline $\begin{array}{l}\text { 50\% cantaloupe+ } \\
\mathbf{5 0 \%} \text { MA extract }\end{array}$ & $\begin{array}{l}23.19^{c} \\
\pm 0.31\end{array}$ & $\begin{array}{l}12.87^{\mathrm{d}} \\
\pm 0.30\end{array}$ & $\begin{array}{l}15.21^{\mathrm{c}} \\
\pm 0.43\end{array}$ & $\begin{array}{l}12.43^{\mathrm{d}} \\
\pm 0.23\end{array}$ & $\begin{array}{l}18.87^{\mathrm{d}} \\
\pm 0.2\end{array}$ & $\begin{array}{l}82.57^{\mathrm{d}} \\
\pm 0.31\end{array}$ \\
\hline
\end{tabular}

a, b, c Mean in the same row are bearing letters, differed significantly at $(\mathrm{P}<0.05)$.

MA: Moringa aqueous.

showed that there were significant differences $(\mathrm{P}<0.05)$ natural antioxidants and total antioxidant activities of different kinds of juice and MA extract.

In Total polyphenols, the highest content was $27.04 \mathrm{mg} / 100 \mathrm{~g} 50 \%$ guava juice $+50 \%$ MA extract, followed by $50 \%$ pomegranate $+50 \%$ MA extract $(26.06 \mathrm{mg} / 100 \mathrm{~g})$, while the lowest content was 20.19 in $50 \%$ cantaloupe $+50 \%$ MA extract. The greatest content of total flavonoids was $17.98 \mathrm{mg} / 100 \mathrm{~g}$ in MA extract, followed by $50 \%$ guava juice $+50 \%$ MA extract $(15.12 \mathrm{mg} / 100 \mathrm{~g})$, while the lowest content was $12.87 \mathrm{mg} / 100 \mathrm{~g}$ in $50 \%$ cantaloupe $+50 \%$ MA extract By observation the results from Table (9) represented the highest content of Ascorbic acid in MA extract $(17.84 \mathrm{mg} / 100 \mathrm{~g})$, followed by $50 \%$ guava juice $+50 \%$ MA extract $(16.02 \mathrm{mg} / 100 \mathrm{~g})$, while the lowest content was $13.34 \mathrm{mg} / 100 \mathrm{~g}$ in $50 \%$ pomegranate $+50 \%$ MA extract. These results were in accordance with those reported by Lee et al., (2012).

On the other hand, data illustrated in Table (9) showed that the highest $\beta$ - carotene content was observed in $50 \%$ guava juice $+50 \%$ MA extract $(15.65 \mathrm{mg} / 100 \mathrm{~g})$, followed by $50 \%$ strawberry juice $+50 \%$ MA extract $(14.41 \mathrm{mg} / 100 \mathrm{~g})$, while the lowest content was $13.59 \mathrm{mg} / 100 \mathrm{~g}$ in MA extract. 
Carotenoids showed that the highest value in MA extract $(27.42 \mathrm{mg} / 100 \mathrm{~g})$, followed by $50 \%$ guava juice $+50 \%$ MA extract $(25.43 \mathrm{mg} / 100 \mathrm{~g}), 50 \%$ cantaloupe $+50 \%$ MA extract recorded the lowest content $(18.87 \mathrm{mg} / 100 \mathrm{~g})$. In Total antioxidant activity, the highest content was $99.26 \mathrm{mg} / 100 \mathrm{~g}$ in $50 \%$ guava juice $+50 \%$ MA extract, followed by MA extract $(98.39 / 100 \mathrm{~g})$, whereas $50 \%$ cantaloupe $+50 \%$ MA extract had the lowest content $(82.57 \mathrm{mg} / 100 \mathrm{~g})$. These results were in accordance with those reported by Venkatachalam et al., (2014)

\section{Sensory evaluation of different fresh fruitjuice as affected by addition of moringa}

Appearance (8), texture (15), color (15), odor (15), flavor (15), taste (20) and overall acceptability (100) are presented in Table 10. Data showed that there were significant differences $(\mathrm{P}<0.05)$ in Sensory evaluation of the formulated fresh juice.

The highest content of appearance was 19.34 MA extract, followed by $50 \%$ cantaloupe $+50 \%$ MA extract, (18.54), whereas the lowest content was 16.87 in $50 \%$ pomegranate $+50 \%$ MA extract, Texture showed that the highest content was 14.54 in $50 \%$ cantaloupe+50\% MA extract, followed by $50 \%$ guava juice $+50 \%$ MA extract (13.21), while the lowest value was 11.52 in MA extract. Color recorded the highest value was 13.98 in $50 \%$ guava juice $+50 \%$ MA extract followed by $50 \%$ cantaloupe+ $50 \%$ MA extract (13.75), whereas the lowest value was 11.72 in MA extract. Odor showed the highest content was 14.64 in $50 \%$ strawberry juice+ 50\% MA extract, followed by MA extract(14.36), while the lowest value was12.34 in 50\% cantaloupe $+50 \%$ MA extract.

On the other hand, data in Table (10), showed that the highest Flavor content was 14.74 in $50 \%$ pomegranate $+50 \%$ MA extract, followed by in 50 $\%$ strawberry juice+ $50 \%$ MA extract (14.53), while the lowest value was 12.43 in MA extract. Taste showed that the highest content was 18.90 in 50\% pomegranate $+50 \%$ MA extract, followed by $50 \%$ cantaloupe $+50 \%$ MA extract (17.83), whereas MA extract had the lowest content (15.31).Overall Acceptability recorded that the highest content was 90.5 in $50 \%$ strawberry juice+ $50 \%$ MA extract, followed by $50 \%$ cantaloupe $+50 \%$ MA extract(89.82), while MA extract had the lowest content (84.68) These results coincide with those reported by Saxena et al., (2014). 
Table (10): Sensory evaluation of different fresh fruitjuice as affected by addition of Moringa aqueous extract

\begin{tabular}{|c|c|c|c|c|c|c|c|}
\hline Content. & $\begin{array}{c}\text { Appearance } \\
\text { (20) }\end{array}$ & $\begin{array}{l}\text { Texture } \\
\text { (15) }\end{array}$ & $\begin{array}{l}\text { Color } \\
\text { (15) }\end{array}$ & $\begin{array}{l}\text { Odor } \\
\text { (15) }\end{array}$ & $\begin{array}{l}\text { Flavor } \\
\text { (15) }\end{array}$ & $\begin{array}{c}\text { Taste } \\
\text { (20) }\end{array}$ & $\begin{array}{c}\text { Overall } \\
\text { Acceptability } \\
\quad(\mathbf{1 0 0})\end{array}$ \\
\hline MA extract & $\begin{array}{l}19.34^{\mathrm{a}} \\
\pm 0.60\end{array}$ & $\begin{array}{l}11.52^{\mathrm{c}} \\
\pm 0.13\end{array}$ & $\begin{array}{l}11.72^{c} \\
\pm 0.79\end{array}$ & $\begin{array}{l}14.36^{\mathrm{a}} \\
\pm 0.08\end{array}$ & $\begin{array}{l}12.43^{b} \\
\pm 0.54\end{array}$ & $\begin{array}{l}15.31^{\mathrm{d}} \\
\pm 0.41\end{array}$ & $\begin{array}{l}84.68^{c} \\
\pm 0.43\end{array}$ \\
\hline $\begin{array}{l}50 \% \text { Strawberry } \\
+\mathbf{5 0 \%} \text { MA extract }\end{array}$ & $\begin{array}{l}18.73^{\mathrm{a}} \\
\pm 0.42\end{array}$ & $\begin{array}{l}12.43^{\mathrm{bc}} \\
\pm 0.61\end{array}$ & $\begin{array}{l}12.54^{\mathrm{b}} \\
\pm 0.04\end{array}$ & $\begin{array}{l}14.64^{\mathrm{a}} \\
\pm 0.32\end{array}$ & $\begin{array}{l}14.53^{\mathrm{a}} \\
\pm 0.23\end{array}$ & $\begin{array}{l}17.63^{\mathrm{b}} \\
\pm 0.20\end{array}$ & $\begin{array}{l}90.5^{\mathrm{a}} \\
\pm 0.67\end{array}$ \\
\hline $\begin{array}{l}\mathbf{5 0 \%} \text { Guava juice } \\
+\mathbf{5 0 \%} \text { MA extract }\end{array}$ & $\begin{array}{c}17.46^{\mathrm{ab}} \\
\pm 0.10 \\
\end{array}$ & $\begin{array}{l}13.21^{\mathrm{b}} \\
\pm 0.52 \\
\end{array}$ & $\begin{array}{l}13.98^{\mathrm{a}} \\
\pm 0.54\end{array}$ & $\begin{array}{l}13.76^{\mathrm{b}} \\
\pm 0.40 \\
\end{array}$ & $\begin{array}{l}12.50^{b} \\
\pm 0.15 \\
\end{array}$ & $\begin{array}{l}16.43^{\mathrm{c}} \\
\pm 0.2\end{array}$ & $\begin{array}{l}87.34^{b} \\
\pm 0.50\end{array}$ \\
\hline $\begin{array}{l}\mathbf{5 0 \%} \text { pomegranate } \\
+\quad \mathbf{5 0 \%} \quad \text { MA } \\
\text { extract }\end{array}$ & $\begin{array}{l}16.87^{\mathrm{c}} \\
\pm 0.64\end{array}$ & $\begin{array}{l}12.52^{\mathrm{bc}} \\
\pm 0.82\end{array}$ & $\begin{array}{l}12.08^{\mathrm{b}} \\
\pm 0.20\end{array}$ & $\begin{array}{l}14.32^{\mathrm{a}} \\
\pm 0.21\end{array}$ & $\begin{array}{l}14.74^{\mathrm{a}} \\
\pm 0.34\end{array}$ & $\begin{array}{l}18.90^{\mathrm{a}} \\
\pm 0.66\end{array}$ & $\begin{array}{l}89.43^{\mathrm{a}} \\
\pm 0.39\end{array}$ \\
\hline $\begin{array}{l}\text { 50\% cantaloupe } \\
+\mathbf{5 0 \%} \text { MA } \\
\text { extract }\end{array}$ & $\begin{array}{l}18.54^{\mathrm{a}} \\
\pm 0.12\end{array}$ & $\begin{array}{l}14.60^{\mathrm{a}} \\
\pm 0.65\end{array}$ & $\begin{array}{l}13.75^{\mathrm{a}} \\
\pm 0.25\end{array}$ & $\begin{array}{l}12.34^{\mathrm{bc}} \\
\pm 0.53\end{array}$ & $\begin{array}{l}12.76^{\mathrm{b}} \\
\pm 0.72\end{array}$ & $\begin{array}{l}17.83^{\mathrm{b}} \\
\pm 0.23\end{array}$ & $\begin{array}{l}89.82^{\mathrm{a}} \\
\pm 0.80\end{array}$ \\
\hline
\end{tabular}

a, b, c Mean in the same row are bearing letters, differed significantly at $(\mathrm{P}<0.05)$.

MA: Moringa aqueous

The total viable bacterial counts (Log CFU/ml) of moringa aqueous extract with juice mixes through 15 days of storage period at $\left(3^{\circ} \mathrm{C}\right)$

Data in Table (11) showed that microbial count total bacterial count unpasteurized and pasteurized. AS shown in the table, no total bacterial counts were detected in all samples during storage at $\left(3^{\circ} \mathrm{C}\right)$ on unpasteurized and pasteurized at zero time and until the seventh day of storage. After storage for seven day, a few bacterial counts were detected in samples. On the $10^{\text {th }}$ day, the highest total count of unpasteurized was $0.0302 \mathrm{Log} C \mathrm{FU} / \mathrm{ml}$ in $50 \%$ pomegranate $+50 \%$ MA extract, followed by $50 \%$ strawberry juice $+50 \%$ MA extract $(0.025 \mathrm{Log} \mathrm{CFU} / \mathrm{ml})$, while the lowest in total was 0.00 in MA extract, on the $15^{\text {th }}$ day, the highest total count of unpasteurized was $0.04 \mathrm{Log}$ $\mathrm{CFU} / \mathrm{ml}$ in $50 \%$ pomegranate juice $+50 \%$ MA extract, whereas MA extract had the lowest in total bacterial count. The findings agree with those of Combina et al., (2007). 
Table (11): The total viable bacterial counts (Log CFU/ml) of moringa aqueous extract with juice mixes through 15 days of storage period at $\left(3^{0} \mathrm{C}\right)$

\begin{tabular}{|c|c|c|c|c|c|c|}
\hline Time & \multicolumn{2}{|c|}{$\begin{array}{c}\text { Initial } \\
\text { day }\end{array}$} & $\begin{array}{l}3^{\text {rd }} \\
\text { day }\end{array}$ & $\begin{array}{l}7^{\text {th }} \\
\text { day }\end{array}$ & $\begin{array}{l}10^{\text {th }} \\
\text { day }\end{array}$ & $\begin{array}{l}15^{\text {th }} \\
\text { day }\end{array}$ \\
\hline \multirow[t]{2}{*}{ MA extract } & up & $\begin{array}{r}0.00 \\
\pm 0.0 \\
\end{array}$ & $\begin{array}{r}0.00 \\
\pm 0.00 \\
\end{array}$ & $\begin{array}{r}0.00^{\mathrm{b}} \\
+0.00 \\
\end{array}$ & $\begin{array}{l}0.00^{\mathrm{c}} \\
\pm 0.00 \\
\end{array}$ & $\begin{array}{l}0.001^{\mathrm{bc}} \\
\pm 0.00 \\
\end{array}$ \\
\hline & $\mathrm{P}$ & $\begin{array}{r}0.00 \\
+0.00\end{array}$ & $\begin{array}{r}0.00 \\
+0.00 \\
\end{array}$ & $\begin{array}{r}0.00^{\mathrm{b}} \\
+0.00 \\
\end{array}$ & $\begin{array}{l}0.00^{\mathrm{c}} \\
+0.0\end{array}$ & $\begin{array}{r}0.00^{\mathrm{c}} \\
+0.00 \\
\end{array}$ \\
\hline \multirow[t]{2}{*}{$\begin{array}{l}50 \% \text { Strawberry } \\
\text { juice+ } 50 \% \text { MA } \\
\text { extract }\end{array}$} & up & $\begin{array}{r}0.00 \\
+.00 \\
\end{array}$ & $\begin{array}{r}0.00 \\
+0.00 \\
\end{array}$ & $\begin{array}{r}0.0021^{\mathrm{a}} \\
+.00 \\
\end{array}$ & $\begin{array}{l}0.025^{\mathrm{a}} \\
\pm 0.00\end{array}$ & $\begin{array}{l}0.0321^{\mathrm{a}} \\
+.00\end{array}$ \\
\hline & $\mathrm{P}$ & $\begin{array}{r}0.00 \\
+0.0\end{array}$ & $\begin{array}{r}0.00 \\
+0.00 \\
\end{array}$ & $\begin{array}{r}0.00^{\mathrm{b}} \\
+0.00 \\
\end{array}$ & $\begin{array}{r}0.00^{\mathrm{c}} \\
+0.00 \\
\end{array}$ & $\begin{array}{l}0.0202^{\mathrm{b}} \\
+.00\end{array}$ \\
\hline \multirow[t]{2}{*}{$\begin{array}{l}50 \% \text { Guava juice+ } \\
50 \% \text { MA extract }\end{array}$} & up & $\begin{array}{r}0.00 \\
+0.0 \\
\end{array}$ & $\begin{array}{r}0.00 \\
+0.00 \\
\end{array}$ & $\begin{array}{r}0.002^{\mathrm{a}} \\
+.00 \\
\end{array}$ & $\begin{array}{r}0.0102^{\mathrm{b}} \\
+0.00 \\
\end{array}$ & $\begin{array}{r}0.0403^{\mathrm{a}} \\
+00 \\
\end{array}$ \\
\hline & $\mathrm{P}$ & $\begin{array}{r}0.00 \\
+0.0\end{array}$ & $\begin{array}{r}0.00 \\
+0.00 \\
\end{array}$ & $\begin{array}{l}0.00^{\mathrm{b}} \\
+0.00 \\
\end{array}$ & $\begin{array}{c}0.0013^{\mathrm{bc}} \\
+0.0\end{array}$ & $\begin{array}{l}0.0111^{\mathrm{b}} \\
+.00\end{array}$ \\
\hline \multirow[t]{2}{*}{$\begin{array}{l}50 \% \text { pomegranate+ } \\
50 \% \text { MA extract }\end{array}$} & up & $\begin{array}{r}0.00 \\
+0.0\end{array}$ & $\begin{array}{c}0.00 \\
\pm 0.00\end{array}$ & $\begin{array}{l}0.001^{\mathrm{b}} \\
+0.00\end{array}$ & $\begin{array}{c}0.0302^{\mathrm{a}} \\
\pm 0.0\end{array}$ & $\begin{array}{c}0.0421^{\mathrm{a}} \\
+01\end{array}$ \\
\hline & $\mathrm{P}$ & $\begin{array}{r}0.00 \\
+0.0\end{array}$ & $\begin{array}{c}0.00 \\
\pm 0.00\end{array}$ & $\begin{array}{l}0.00^{\mathrm{b}} \\
+0.00\end{array}$ & $\begin{array}{l}0.00^{\mathrm{c}} \\
+0.00 \\
\end{array}$ & $\begin{array}{l}0.0011^{\mathrm{bc}} \\
\pm 0.0\end{array}$ \\
\hline \multirow[t]{2}{*}{$\begin{array}{l}\text { 50\% cantaloupe+ } \\
50 \% \text { MA extract }\end{array}$} & up & $\begin{array}{r}0.00 \\
+0.0 \\
\end{array}$ & $\begin{array}{r}0.00 \\
+0.00 \\
\end{array}$ & $\begin{array}{l}0.00^{\mathrm{b}} \\
+.00 \\
\end{array}$ & $\begin{array}{r}0.0200^{\mathrm{a}} \\
+.00 \\
\end{array}$ & $\begin{array}{r}0.0210^{\mathrm{b}} \\
+00 \\
\end{array}$ \\
\hline & $\mathrm{P}$ & $\begin{array}{r}0.00 \\
+.00\end{array}$ & $\begin{array}{r}0.00 \\
+0.00\end{array}$ & $\begin{array}{l}0.00^{\mathrm{b}} \\
+0.00\end{array}$ & $\begin{array}{r}0.00^{\mathrm{c}} \\
+0.00\end{array}$ & $\begin{array}{l}0.002^{\mathrm{bc}} \\
\pm 0.00\end{array}$ \\
\hline
\end{tabular}

Up: Un pasteurized - P: Pasteurized MA: Moringa aqueous

Yeasts and molds count (Log CFU/ml) of moringa aqueous extract with juice mixes through 15 days of storage period at $\left(3^{\circ} \mathrm{C}\right)$

Data in Table (12) Yeasts and molds count of: unpasteurized and pasteurized were carried out before the storage and after storage for fifteen days. The results were recorded in Table (12).

No yeasts or molds count were detecting in all samples during storage $\left(3^{0} \mathrm{C}\right)$ until tenth day. On the $15^{\text {th }}$ day, the pomegranate moringa mix recorded $(0.0035 \mathrm{Log} \mathrm{CFU} / \mathrm{ml})$, followed by strawberry moringa mix 
Table (12): Yeast and mold counts (Log CFU/ml) of moringa aqueous extract with juice mixes through 15 days of storage period at $\left(3^{0} \mathrm{C}\right)$.

\begin{tabular}{|c|c|c|c|c|c|c|}
\hline \multirow{2}{*}{$\begin{array}{l}\text { Time } \\
\text { Sample }\end{array}$} & \multicolumn{2}{|c|}{ Zero day } & $3^{\text {rd }}$ day & $7^{\text {th }}$ day & $10^{\text {th }}$ day & $15^{\text {th }}$ day \\
\hline & \multicolumn{2}{|c|}{ Y\&M } & Y\&M & Y\&M & Y\&M & Y\&M \\
\hline \multirow[t]{3}{*}{ MA extract } & up & 0.00 & 0.00 & 0.00 & 0.00 & $0.00^{c}$ \\
\hline & & \pm 0.00 & \pm 0.00 & \pm .00 & \pm 0.00 & \pm 0.00 \\
\hline & $\mathrm{P}$ & $\begin{array}{r}0.00 \\
+0.00\end{array}$ & $\begin{array}{r}0.00 \\
+0.00 \\
\end{array}$ & $\begin{array}{r}0.00 \\
+0.00 \\
\end{array}$ & $\begin{array}{r}0.00 \\
+0.00 \\
\end{array}$ & $\begin{array}{l}0.001^{\mathrm{bc}} \\
\pm 0.00\end{array}$ \\
\hline \multirow[t]{2}{*}{$\begin{array}{l}50 \% \text { Strawberry } \\
\text { juice+ } \\
50 \% \text { MA extract }\end{array}$} & up & $\begin{array}{r}0.00 \\
+0.00 \\
\end{array}$ & $\begin{array}{r}0.00 \\
+0.00 \\
\end{array}$ & $\begin{array}{r}0.00 \\
+.00\end{array}$ & $\begin{array}{r}0.00 \\
+0.00 \\
\end{array}$ & $\begin{array}{r}0.0027^{\mathrm{a}} \\
+0.0 \\
\end{array}$ \\
\hline & $\mathrm{P}$ & $\begin{array}{r}0.00 \\
+000\end{array}$ & $\begin{array}{r}0.00 \\
+0.00 \\
\end{array}$ & $\begin{array}{r}.0 .00 \\
+0.0\end{array}$ & $\begin{array}{r}0.00 \\
+0.00 \\
\end{array}$ & $\begin{array}{l}0.000^{\mathrm{c}} \\
\pm 0.00\end{array}$ \\
\hline \multirow[t]{2}{*}{$\begin{array}{l}50 \% \text { Guava juice+ } \\
50 \% \text { MA } \\
\text { extract }\end{array}$} & up & $\begin{array}{r}0.00 \\
+.00\end{array}$ & $\begin{array}{r}0.00 \\
+0.00 \\
\end{array}$ & $\begin{array}{r}0.00 \\
+0.00 \\
\end{array}$ & $\begin{array}{r}0.00 \\
+0.00 \\
\end{array}$ & $\begin{array}{l}0.002^{\mathrm{b}} \\
+0.00\end{array}$ \\
\hline & $\mathrm{P}$ & $\begin{array}{r}0.00 \\
+0.00 \\
\end{array}$ & $\begin{array}{r}0.00 \\
+.00\end{array}$ & $\begin{array}{r}0.00 \\
+0.00 \\
\end{array}$ & $\begin{array}{r}0.00 \\
+0.00 \\
\end{array}$ & $\begin{array}{r}0.00^{\mathrm{c}} \\
+0.00 \\
\end{array}$ \\
\hline \multirow[t]{2}{*}{$\begin{array}{l}50 \% \\
\text { pomegranate+ } \\
50 \% \text { MA extract }\end{array}$} & up & $\begin{array}{r}0.00 \\
+0.00\end{array}$ & $\begin{array}{r}0.00 \\
+0.00 \\
\end{array}$ & $\begin{array}{r}0.00 \\
+.00\end{array}$ & $\begin{array}{r}0.00 \\
+0.00 \\
\end{array}$ & $\begin{array}{r}0.0035^{\mathrm{a}} \\
+0.00 \\
\end{array}$ \\
\hline & $\mathrm{P}$ & $\begin{array}{r}0.00 \\
+0.00\end{array}$ & $\begin{array}{c}0.00 \\
+0.00\end{array}$ & $\begin{array}{r}0.00 \\
+0.0\end{array}$ & $\begin{array}{r}0.00 \\
+0.00\end{array}$ & $\begin{array}{r}0.00^{\mathrm{c}} \\
+0.00\end{array}$ \\
\hline \multirow[t]{2}{*}{$\begin{array}{l}50 \% \text { cantaloupe+ } \\
50 \% \text { MA extract }\end{array}$} & up & $\begin{array}{r}0.00 \\
+0.00\end{array}$ & $\begin{array}{r}0.00 \\
+0.0 \\
\end{array}$ & $\begin{array}{r}0.00 \\
+0.0 \\
\end{array}$ & $\begin{array}{c}0.00+ \\
0.00\end{array}$ & $\begin{array}{l}0.001^{\text {bc }} \\
+0.00\end{array}$ \\
\hline & $\mathrm{P}$ & $\begin{array}{r}0.00 \\
+00 \\
\end{array}$ & $\begin{array}{r}0.00 \\
+0.00 \\
\end{array}$ & $\begin{array}{r}0.00 \\
+.00\end{array}$ & $\begin{array}{r}0.00 \\
+0.00\end{array}$ & $\begin{array}{r}0.00^{\mathrm{c}} \\
+0.00\end{array}$ \\
\hline
\end{tabular}

Up: unpasteurized - p: pasteurized, MA: Moringa aqueous, Y: yeast, M: mold

(0.0027Log CFU/ml), while Moringa aqueous extract had no detected in yeast and molds count $(0.00 \mathrm{Log} \mathrm{CFU} / \mathrm{ml})$, The findings agree with those of Dangana,et al.,(2013).

\section{Coliform count (Log CFU/ml) of moringa aqueous extract with juice} mixes through 15 days of storage period at $\left(3^{\circ} \mathrm{C}\right)$ :

All results on zero day, $3 \mathrm{rd}$ day, $7^{\text {th }}$ day, $10^{\text {th }}$ day and finally $15^{\text {th }}$ day are (0.00), and in all groups as shown in Table 13 .The finding agree with those of Goja and Mohamed. (2013). 
Table (13): Coliform count ( $\log \mathrm{CFU} / \mathrm{ml})$ of moringa aqueous extract with juice mixes through 15 days of storage period at $\left(3^{0} \mathrm{C}\right)$

\begin{tabular}{|c|c|c|c|c|c|c|}
\hline $\begin{array}{ll}\text { Sample of } \\
\text { blends }\end{array}$ & & $\begin{array}{l}\text { Zero } \\
\text { day }\end{array}$ & $\begin{array}{l}3^{\text {rd }} \\
\text { day }\end{array}$ & $\begin{array}{c}7^{\text {th }} \\
\text { day }\end{array}$ & $\begin{array}{l}10^{\text {th }} \\
\text { day }\end{array}$ & $\begin{array}{l}15^{\text {th }} \\
\text { day }\end{array}$ \\
\hline \multirow[t]{2}{*}{ MA extract } & $\mathrm{F}$ & 0.00 & 0.00 & 0.00 & 0.00 & 0.00 \\
\hline & $\mathrm{P}$ & 0.00 & 0.00 & 0.00 & 0.00 & 0.00 \\
\hline \multirow{2}{*}{$\begin{array}{l}50 \% \text { Strawberry juice+ } \\
50 \% \text { MA extract }\end{array}$} & $\mathrm{F}$ & 0.00 & 0.00 & 0.00 & 0.00 & 0.00 \\
\hline & $\mathrm{P}$ & 0.00 & 0.00 & 0.00 & 0.00 & 0.00 \\
\hline \multirow{2}{*}{$\begin{array}{l}\mathbf{5 0 \%} \text { Guava juice+ } \\
\mathbf{5 0 \%} \text { MA extract }\end{array}$} & $\mathrm{F}$ & 0.00 & 0.00 & 0.00 & 0.00 & 0.00 \\
\hline & $\mathrm{P}$ & 0.00 & 0.00 & 0.00 & 0.00 & 0.00 \\
\hline \multirow{2}{*}{$\begin{array}{l}50 \% \text { pomegranate }+ \\
50 \% \text { MA extract }\end{array}$} & $\mathrm{F}$ & 0.00 & 0.00 & 0.00 & 0.00 & 0.00 \\
\hline & $\mathrm{P}$ & 0.00 & 0.00 & 0.00 & 0.00 & 0.00 \\
\hline \multirow{2}{*}{$\begin{array}{l}50 \% \text { cantaloupe+ } \\
50 \% \text { MA extract }\end{array}$} & $\mathrm{F}$ & 0.00 & 0.00 & 0.00 & 0.00 & 0.00 \\
\hline & $\mathrm{P}$ & 0.00 & 0.00 & 0.00 & 0.00 & 0.00 \\
\hline
\end{tabular}

F: Refrigerator, P: Pasteurization 0: No detected, MA: Moringa aqueous Extract.

Conclusively, from obtained results, It can strengthen the Moringa aqueous extract with fruit juices to raise the nutritional value and as an antioxidant and antimicrobial (as a natural preservative).

\section{REFRENCES}

Akther, S.F; Shahriar, M.S; Morshed,M.M; and. Islam, M. N. (2012):Study on Chemical Composition of Fresh Mymensingh and Barishal HogplumSpondiusmangifera) and Developed Leather and Jelly and Sensory Evaluation J. Environ. Sci. \& Natural Resources, 5(2): 29 - 36.

Al-Dabbas, M;Ahmad, R; Radway,Y. and Abulaila, K. (2010):Chemical composition and oil components in seeds of Moringa peregrina (Forssk) Fiori. Crop Res., 40 (1, 2 \& 3): 161-167 with one figure Printed in India.

Al-Kharusi, L.M; Elmardi, M.O; Ali, A ; Al-Said, F.A.J; Abdelbasit; K.M and Al-Rawahi, S. (2009): Effect of mineral and organic fertilizers on the emical characteristics and quality of date fruits . Int. J. Agric Biol.,11: 290296.

Alothman, M.; Bhat, R; and Karim, A. A. (2009):Antioxidant capacity and phenolic content of selected tropical fruits from Malaysia, extracted with different solvents, Food Chemistry, 115: 785-788.

A.OA.C. (2005):Official Methods of Analysis of the Association of Official Agricultural Chemists Published by the association of official Analytical Chemists Washington 25,D.C.,U.S.A. 
Arun, K.; Verma, .V; Rajkumar, R; Banerjee, S.; and Arun, K.(2013): Guava (Psidiumguajava L.) Powder as an Antioxidant Dietary Fibre in Sheep Meat Nuggets-Asian-Australas J. Anim Sci. , 26(6): 886-895.

Awsi, J; and Masih, G.(2012):Development and Quality Evaluation of Pineapple Juice Blend with Carrot and Orange juice. International Journal of Scientific and Research Publications ISSN, 2250-3153,

Broin, M; (2006): The nutrient value of Moringa oleifera Lam. leaves:t can we learn from figure? Moringa news work shop. http: //www. moringanews. org/doc/GB? Posters? Asian Journal of Plant Science and Research, 5(2):8-16.

Busani, M.; Patrick, J. ; Arnold, H; and Voster, M. (2011): Nutritional characterization of Moringa oleifera Lam.) Leaves, African Journal of Biotechnology, 10(60): 12925-12933.

Chuku, L. C and Chinaka, N. C. (2014):Protein and mineral element levels of some fruit juices (Citrus spp.) in some Niger Delta areas of NigeriaInternational Journal of Nutrition and Food Sciences, 3(6-1): 5860.

Combina, M ; Daguerre, C; Masser.A; Mercado, M. E.; Sturm, A. and Martinez, C. (2007) :Yeast identification in grape juice concentrates from Argentina- International Journal of Nutrition and Food Sciences,3(6-1): 58-60.

Coppin, J. (2014):Astudy of the nutritional and medical values of Moringa oleifera leaves from sub-saharan Africa: Ghana, Rwanda ,Senegal and Zambia. J. Sci. Food and Agric., 80- 2027-2021.

Dangana, A ; Mohammed,M.A, ; Rubainu, M. G; Adam, F.I; Emelike, F.O. and Kurue,U.J;(2013):Bacteriological assessment of guava and orange fruits in north east Nigeria-Innovations in Pharmaceuticals and Pharmacotherapy-69, 21-25

El - Massry, Fatma; H. M., Mossa, M. E. M and Youssef, S. M. (2013): Moringa oleifera Plant "Value and Utilization in Food Processing. Egypt. J. Agric. Res., 91 (4), 155-177.

El-sherif, G. and Alghamdi, E. (2013):Influence of Drying Methods on Physicochemical Constituents of Guava. Journal of American Scienc $327,1-2,12-16$.

Fakurazi, S. Sharifudin, S. A. and Arulselvan, P. (2012). Moringa oleifera Hydroethanolic Extracts Effectively Alleviate Acetaminophen-Induced Hepatotoxicity in Experimental Rats through Their Antioxidant NatureMolecules, 17: 8334-8350. 
Gaikwad,M; Kale, S; Bhandare, S.; Urunkar, V and Rajmane, A. (2011):Extraction, characterization and comparison of fixed oil of Moringaoleifera $\mathrm{L}$ and Moringaconcanensisimmofam.Moringaceae. International Journal of PharmTech Research Coden (USA): IJPRIF ISSN: 0974-4304, 3(3) 1567-1575,

Giampieri, D.S.; Tulipani, S.F.; Alvarez-Suarez, J. M. and Quiles, L. J. (2012).The strawberry: Composition, nutritional quality, and impact on human health. Journal of Nutritional, volume 28-Issue1-132-154.

Gimeno, A..I; Castellote, R.M;.andLamuela-Raventos, M.C. (2000):Rapid determination of vitamin $\mathrm{E}$ in vegetable oils by reversedphase highperformance liquid chromatography-Journal of Chromatography A, 881 251-254.

Goja,A.M; and Mohamed, S. O. (2013): Preliminary Study on Efficacy of Leaves, Seeds and Bark Extracts of Moringaoleifera in Reducing Bacterial load in Water - International Journal of Advanced Research, Volume 1, Issue, 4: 124-130.

Hemalatha, H. and. Anbuselvi, S. (2013):Physicochemical constituents of pineapple pulp and waste- Journal of Chemical and Pharmaceutical Research, 323, 7: 439-445.

Howell, A.B. and D'Souza, D. H. (2013):The Pomegranate: Effects on Bacteria and Viruses. That Influence Human Health-Evid Based Complement Alternat Med, 5:212-216

Jasmine, Y. (2012): Comparison of Sugar Content in Bottled 100\% Fruit Juice versus Extracted Juice of Fresh Fruit - Food and Nutrition Sciences, 3: 1509-1513.

Karol, W; Tadeusz,B;and Jan. K;(2012):Factors affecting the chemical composition of strawberry fruits-polish journal of natural sciences -Abbrev. Pol. J. Natur. Sc., 27(1): 5-13.

Khattab, M. M. A. and Shak, M. A. S. (2012): Effect of frying on the Physicochemical properties of Moringa oleifera, groundnut (Arachishypogaea) ail and their Blends. Journal of Science and Technology, Agricultural and Veterinary Sciences (JAVS No. 2), 7:111-124.

Lallianrawna, S; Muthukumaran, R. and Vanlalhruaii, R. G. (2013): Determination of total phenolic content, total flavonoid content and total antioxidant capacity of Ageratinaadenophora (Spreng.) King \& H. RobSci Vis, Vol 13 Issue No 4 -October-DISSN (print) 0975-6175

Lee,J.H; Lee,Y.B; Woo Duck Seo, W. D; Kang, S.T; Lim, J.W; and Cho, K. M.(2012):Comparative Studies of Antioxidant Activities and Nutritional Constituents of Persimmon Juice (Diospyros kaki L. cv. Gapjubaekmok), Prev. Nutr. Food Sci. Jun, 17(2): 141-151. 
Leghari, M. H.; Sheikhs, J.M.; Memon, N. N. and. Hooharo, A. A.(2013):Quality Attributes of Immature Fruit of Different Mango Varieties- Journal of Basic \& Applied Sciences, 9: 52-56.

Mahendran, T.(2010). Physico-chemical properties and sensory characteristics of dehydrated guava concentrate: effect of drying method and maltodextrin concentration- $t$ mahendran : quality of instant guava drink powder. New England Journal of Medicine, 7:277-289

Mahamood, K.T.; Mugal, T. and Ul Haq, I. (2010):Moringa oleifera: a natural gift. A review- Khawaja Tahir Mahmood et al, J. Pharm. Sci. \& Res., .2 (11): 146-176.

Mariäa, M.; Gil, F; Tomaä,S;Betty, H. and Kader, A. (2002):Antioxidant capacities, phenolic compounds, carotenoids, and Vitamin C Contents of Nectarine, Peach, and Plum Cultivars from California-J. Agric. Food Chem., 50:4976-4982

Miano,T.F,Jokhio,J.A, and Miano, T. F. (2010):Effect of different packaging materials and storage conditions on physico-chemical characteristics of guava (var.Allahabadi). J. Agrofor. Environ., 4 (2): 33-36, 2010.

Mogobe, K.; Mosepele , G. and Masamba, M. (2015). Essential mineral content of common fish species in chanoga, okavango Delta, BotswanaAfrican Journal of Food Science, 128:79-84.

Muthukumar, M; Naveena, B. M. ; Vaithiyanathan, S.; Sen. A. R. and Sureshkumar, K.(2014). Effect of incorporation of Moringa oleifera leaves extract on quality of ground pork patties-J . Food Sci. Technol., 105: $1382-1389$.

Ndong, M. S. and Wade, N. (2007). Nutritional value of Moringa oleifera, study of the bioavailability of iron and the effect of enrichment of various traditional senegalese meals with powder of the leaves, African Journal of food Agriculture, Nutrition, and development.93, 2, 253-263

Nkafamiya, I. I.; Osemeahon, S. A.; Modibbo, U.U., Aminu, A; (2010): Nutritional tus of non-conventional leaf vegetables. ficu sas peri folia and Ficussycomorus. Afr. J. Food Sci., 4(3): 104-108.

Notter, G. K; Taylor, P.H. and Downens , N. J. (1995): Orange juice powder Factors affecting storage stability. Food Tech.,13,113. Academia Arena

Ogunbanwo, S. T; Azinat, S; and Fadahunsi, I. F.(2013):Microbiological And Nutritional Evaluation Of Water Melon Juice (Citrulluslanatus), Academia Arena, 5(3) volume(12)133-177.

Ranganna, S.I. (1999): Manual of Analysis of fruit and vegetables products, Ascorbic acid.pp94-96.Tata Mc Grew-Hill publishing Company 96 Tata Mc Grew-Hill Pu Company Limited, New Delhi., 634. 
Saxena, T. M; Raju, P.S; and. Bawa, A. S. (2014):Evaluation of sensory acceptability and storage stability of frozen carrot based dessert-Journal of Food Sci. Technol. Jun, 51(6): 1203-1207

Seshadri, S. and Nambiar, V. (2003).Digeraarvensis and drumstick leaves Moringa oleifera): Nutrient profile and potential for human consumption. In Plants in Human Health and Nutrition Policy, J. Clin. Nut., 211, 52-55

Shahnawaz, M.; Sheikh, S.A. and Hussain, M. (2013):A study on the determination of physicochemical properties of honey from different valleys of Gilgit- Baltistan. International Journal of Agricultural Science Research, 2(2), pp. 049-053,

Shahriar, M; Hossain, I; Bahar, A.N. and Akhter, S. (2012):Preliminary Phytochemical Screening, In-Vitro Antioxidant and Cytotoxic Activity of Five Different Extracts of Moringa oleifera Leaf. Journal of Applied Pharmaceutical Science 02 (05); 65-68.

Singleton,V.L. and Rossi , J.A. (2005):Colorimetetric determination of total phenol with Phosphomolybdic acid reagents.-American Journals of Enology Viticulture,16.144-158. International Journal of food Science and Technology 37.153-161.

Singh, C. S; and Bunkar, D. S. (2005). Optimization of Nutritional Drink of Pomegranate, Orange and Ginger Juices using Response Surface Methodology-Food Processing \& Technology, 49: 3101-3105

Smith, F.; Gilles, M.A; Haniltun, J. K. and Geodes, A.A. (1999). Colorimetric methods for determination of sugar and related sugar and related substances. Anal.Chem., 211-255-55.

Steel, R.G and Torrie, T.H. (1980). Principles And Procedures Of Tatistics: a Biometrical Approach Second Edition. New York: M cGraw-ill Kogakusha.

Tanwar,B; Andallu, B; and Chandel, S;(2014) :Influence of processing on physicochemical and nutritional composition of psidium guava L. (Guava) Products. International Journal of Agriculture and Food Science Technology, ISSN 2249-3050, 5( 2): 47-54

Venkatachalam, K., Rangasamy, R. and Krishnan, V. (2014): Total antioxidant activity and radical scavenging capacity of selected fruits and vegetables from South India-International Food Research Journal 21(3): 1039-1043.

Warakaulle, S.T.S.K.; Weerathilake, W. A. D.V. and Abeynayake, N.R.(2014). Production and Evaluation of Set type Yogurt Incorporated with Water melon (Citralluslanatus)International Journal of Scientific and Research Publications, Volume 4, Issue 10, 49: 3101-3105 
Wettestein, D.V.(1997): Chlorophyll 1 tale and der Up mikro Skopische from weckses der plastiden Expert. J. Agric. Food Chem., 49: 3101-3105

\section{دراسات كيميائية وميكروييولوجية على المستخلص المائى لاوراق المورينجاوتاثير اضافتها على عصائر الفاكهة}

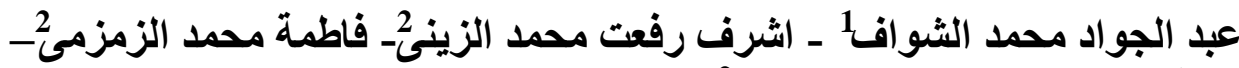 نبيلة محمد سبد الجمد محمد الثواف عمد موض

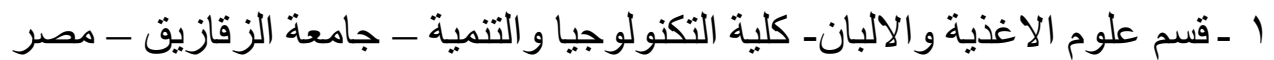

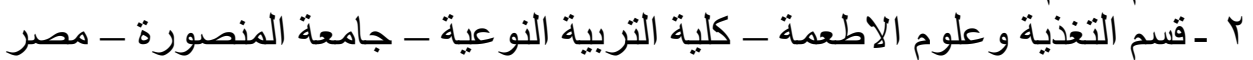

$$
\begin{aligned}
& \text { تهذف هذة الدر اسة الى توضيح اثر اضافة المستخلص المائى لاوراق } \\
& \text { المورينجا لعصائر الفاكهة الطازجة على الخواس الكيميائية والميكروبية } \\
& \text { و الفسيولوجية و الحسية. }
\end{aligned}
$$

حيث اظهرت النتائج ان: المستخلص المائى لاوراق المورينجا ذو قيمة وظئه وظيفية

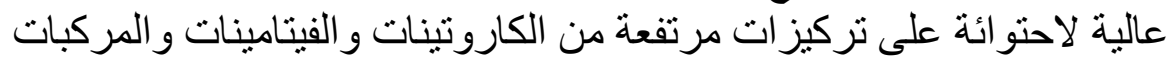

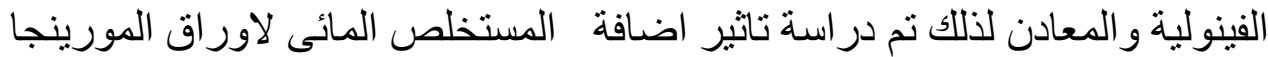

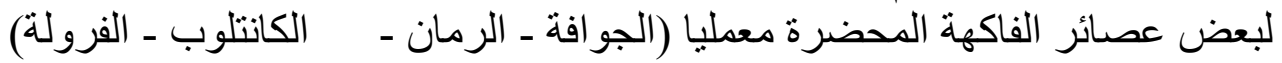

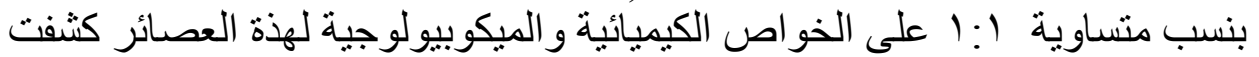

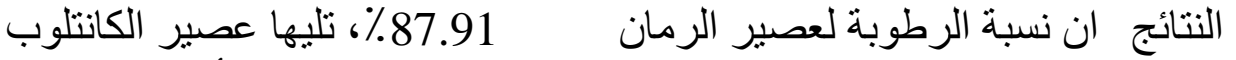

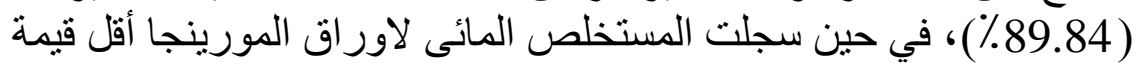

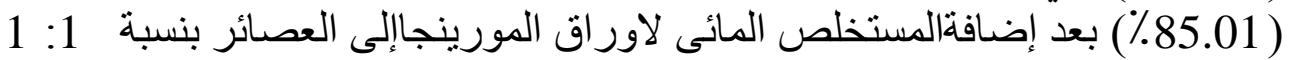

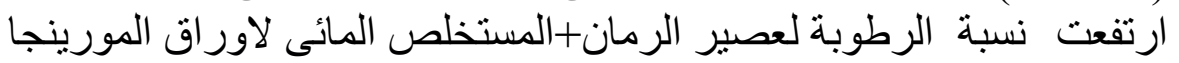

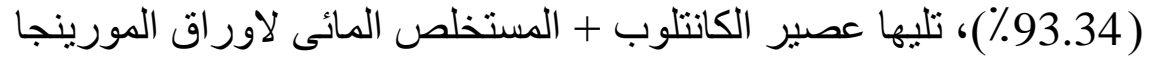

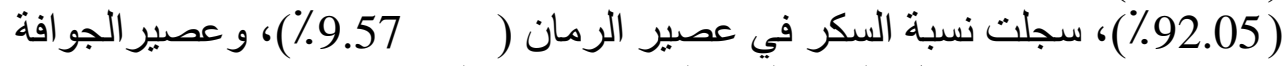

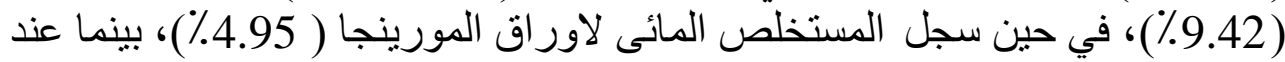

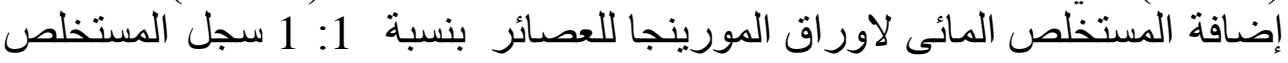

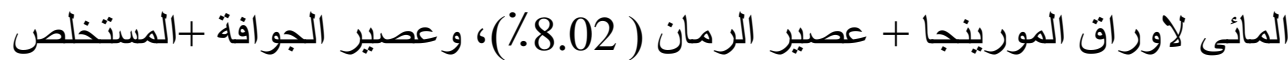
المائى لاوراق المورينجات المورينا + 7.91\%).

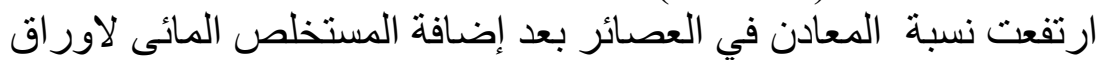

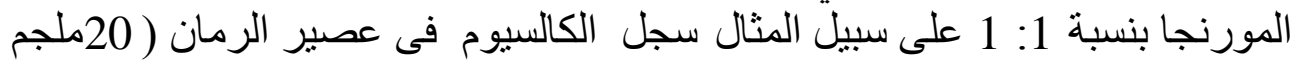




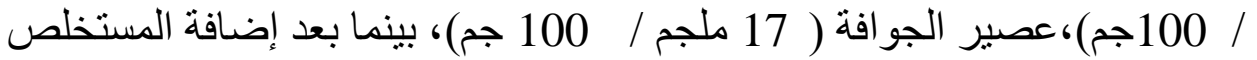

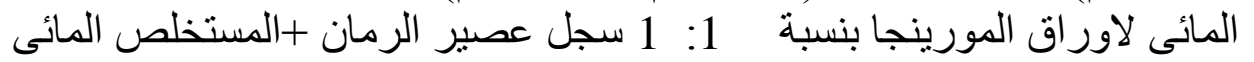

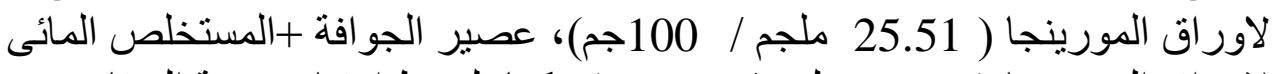

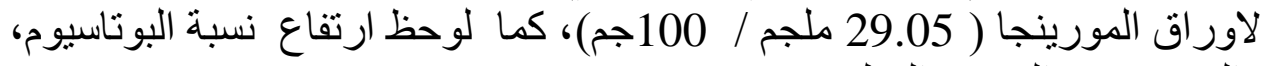

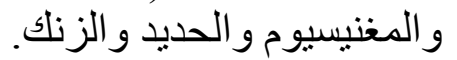

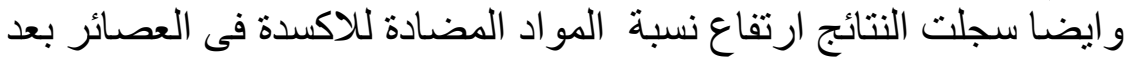

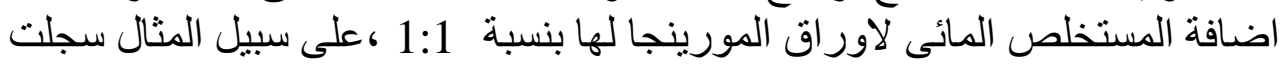

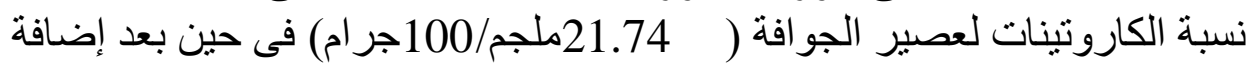

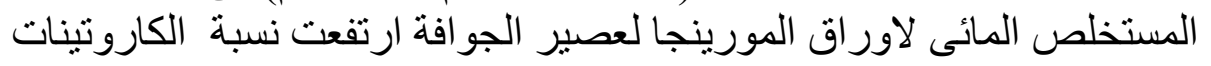

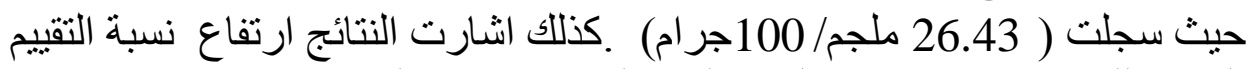

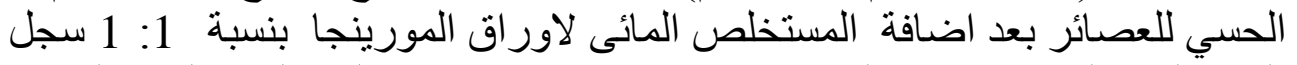

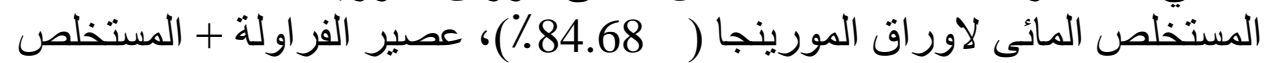

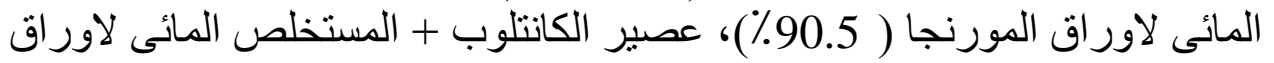

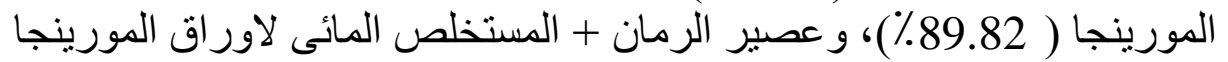

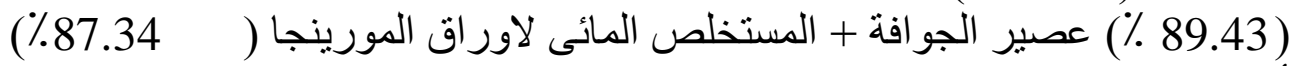

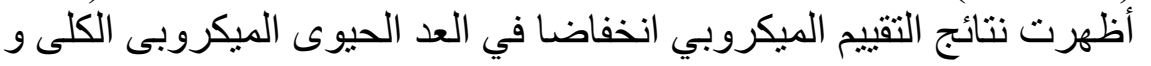

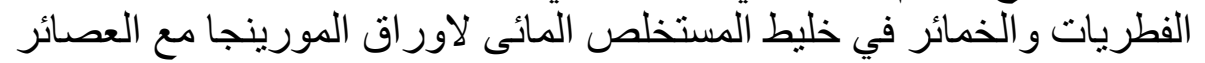

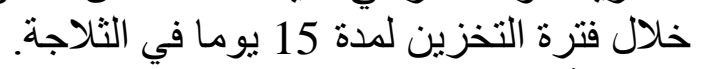

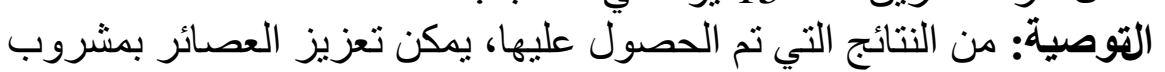

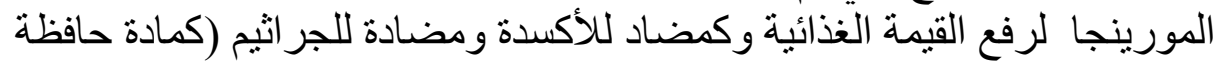
طبيعية). الكلمات الدالة: المستخلص المائى لاوراق المورينجا ، ومضادات الأكسدة، مضادات

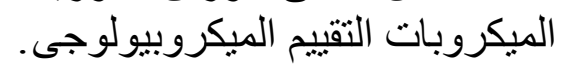

